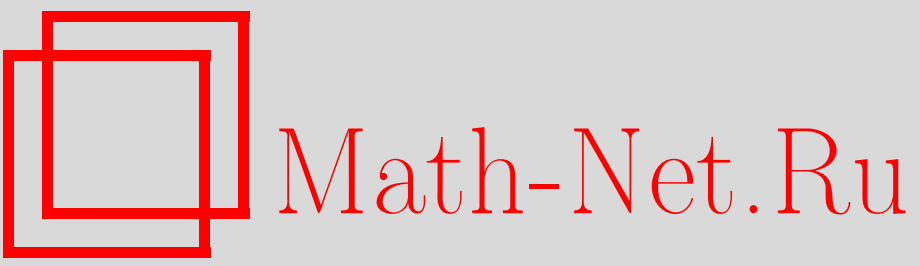

А. М. Райгородский, Проблема Борсука и хроматические числа некоторых метрических пространств, УМН, 2001, том 56, выпуск 1, 107-146

DOI: https://doi.org/10.4213/rm358

Использование Общероссийского математического портала Math-Net.Ru подразумевает, что вы прочитали и согласны с пользовательским соглашением

http://www.mathnet.ru/rus/agreement

Параметры загрузки:

IP : 18.234 .197 .8

26 апреля 2023 г., 16:02:26 


\title{
ПРОБЛЕМА БОРСУКА И ХРОМАТИЧЕСКИЕ ЧИСЛА НЕКОТОРЫХ МЕТРИЧЕСКИХ ПРОСТРАНСТВ
}

\begin{abstract}
А. М. РАЙГОРОДСКИЙ
В настоящей работе дан подробньй обзор различных результатов, касающихся двух известных задач комбинаторной геометрии: задачи Борсука о разбиении произвольного ограниченного $d$-мерного множества ненулевого диаметра на части меньшего диаметра и проблемы отыскания хроматических чисел некоторых метрических пространств. Кроме того, в работе описан некоторьй общий метод, позволяющий получать хорошие нижние оценки как для минимального числа частей меньшего диаметра, на которые разбивается любое ограниченное неодноточечное множество размерности $d$, так и для хроматических чисел различных метрических пространств, - в частности, для $\mathbb{R}^{d}$ и для $\mathbb{Q}^{d}$. Наконец, в задаче об оценке хроматических чисел сформулированы и доказаны новые нижние оценки в некоторых малых размерностях, а также предложены новые естественные обобщения понятия хроматического числа пространства.

Библиография: 104 названия.
\end{abstract}

\section{СОДЕРЖАНИЕ}

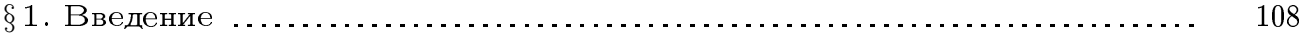

$\S 2$. Проблема Борсука и различные подходы к ее решению ............. 109

2.1. Решение проблемы Борсука в "малых" размерностях ............... 109

2.2. Решение проблемы Борсука для некоторых специальных классов мно-

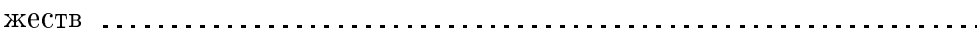

2.3. Верхние оценки на минимальное число частей меньшего диаметра .....

2.4. Контрпримеры к гипотезе Борсука и нижние оценки на минимальное число частей меньшего диаметра ............................ 114

$\S 3$. Хроматические числа некоторых метрических пространств ........ 115

3.1. Некоторые предварительные замечания ........................ 115

3.2. Оценки хроматических чисел в "малых" размерностях .............. 116

3.3. Оценки хроматических чисел с ростом размерности и реализация всех расстояний при разбиении пространства $\mathbb{R}^{d}$ на некоторое число частей $\quad 117$

3.4. Одно обобщение понятия хроматического числа пространства . ........ 120

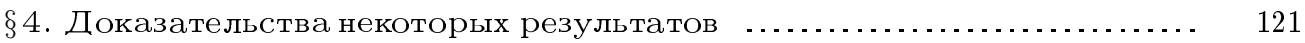

4.1. Оценки величин $\chi\left(\mathbb{Q}^{d}\right)$ и $\chi\left(\mathbb{R}^{d}\right)$ при малых значениях $d \quad \ldots \ldots \ldots . . . . . . . .121$

4.2. Асимптотические оценки величин $f(d), \chi\left(\mathbb{R}^{d}\right), \chi\left(\mathbb{Q}^{d}\right), \chi\left(\mathbb{R}^{d}, a_{1}, \ldots, a_{k}\right) \quad 129$

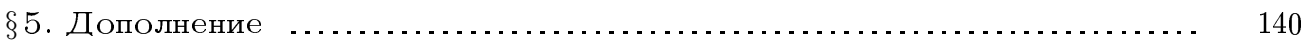

5.1. Проблема Борсука для $(0,1)$-многогранников и кросс-политопов . ..... 140

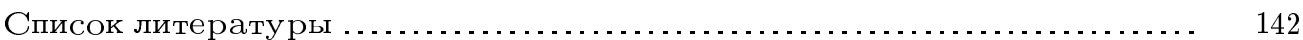

(C) А. М. РАЙГОРОДСКИЙ 2001 


\section{$\S$ 1. Введение}

В 1933 году К. Борсук (см. [1]) высказал гипотезу о том, что всякое ограниченное $d$-мерное точечное множество $\Omega \subset \mathbb{R}^{d}$, имеющее ненулевой диаметр, может быть разбито на $d+1$ часть меньшего диаметра:

$$
\Omega=\Omega_{1} \sqcup \cdots \sqcup \Omega_{d+1}, \quad \operatorname{diam} \Omega_{i}<\operatorname{diam} \Omega \forall i=1, \ldots, d+1 .
$$

Здесь под диаметром множества $\Omega$ понимается величина

$$
\operatorname{diam} \Omega=\sup _{x, y \in \Omega} d(x, y)
$$

где, в свою очередь, $d(x, y)$ - стандартная евклидова метрика.

Немного позднее, в 1944 году, Г. Хадвигер (см. [2]) показал, что если все евклидово пространство $\mathbb{R}^{d}$ произвольньм образом покрыто замкнутыми множествами, число которых в точности равно $d+1$, то хотя бы в одном из этих множеств все неотрицательные вешественные числа реализуются как расстояния между парами точек этого множества. Иными словами, если

$$
\mathbb{R}^{d}=\Omega_{1} \cup \cdots \cup \Omega_{d+1}
$$

и все множества $\Omega_{i}$ замкнутые, то найдется такое $i$, что

$$
\mathbb{R}^{+} \cup\{0\}=\left\{d(x, y): x, y \in \Omega_{i}\right\} .
$$

Наконец, Е. Нелсон и Дж. Р. Исбел, а также, независимо, П. Эрдеш и Г. Хадвигер сформулировали следующую проблему: каково минимальное число цветов, в которые необходимо раскрасить все евклидово пространство $\mathbb{R}^{d}$, так чтобы точки, расстояние между которьми в точности равно единице, оказались раскрашенными в разные цвета? Иначе говоря, чему равна величина $\chi\left(\mathbb{R}^{d}\right)$ - хроматическое число евклидова пространства, - если мы определяем эту величину как хроматическое число бесконечного граф̆а, множество вершин которого совпадает с $\mathbb{R}^{d}$, а множество ребер задается всевозможньми парами точек $x, y \in \mathbb{R}^{d}$, для которых $d(x, y)=1$ ?

На первый взгляд, связь между тремя упомянутыми задачами невелика. Конечно, все они относятся к тому разделу математики, которьй принято называть "комбинаторной геометрией”. Однако одного подобного обстоятельства никак не достаточно для написания такого обзора. Тесная же связь между сформулированными проблемами была установлена лиш совсем недавно: был предложен некоторьй общий метод, позволяющий получать достаточно глубокие результаты как относительно гипотезы Борсука, так и в проблеме отыскания "хроматических чисел евклидова пространства”, причем в последнем случае оказалось возможным эффективно работать не только в ситуации, когда рассматриваемое пространство вешественное $-\mathbb{R}^{d}$, но и в некоторых других случаях, например, в случае рационального пространства $\mathbb{Q}^{d}$ с евклидовой метрикой. Связь же между задачей нахождения хроматического числа и теоремой Хадвигера о реализации всех расстояний и без того непосредственно вытекает из приведенных выше формулировок. В настоящей работе мы постараемся дать подробный

Работа выполнена при финансовой поддержке Российского фонда фундаментальных исследований (гранты № № 99-01-00357, 00-15-96109). 
обзор различных результатов, касающихся этих задач, описать упомянутьй общий метод и доказать несколько новых результатов, связанных, в частности, с применением этого метода.

\section{§ 2. Проблема Борсука и различные подходы к ее решению}

В этом параграфе будет изложена история вопроса и сформулированы различные подходы к получению ответа на этот вопрос.

2.1. Решение проблемы Борсука в “малых" размерностях. Итак, мы уже знаем, что в 1933 году К. Борсук сформулировал следующий вопрос: верно ли, ито всякое ограниченное неодноточечное множество в $\mathbb{R}^{d}$ может бить представлено в виде обвединения своих частей меньшего диаметра, причем так, чтобь количество этих частей не превышало $d+1$ ? (См. [1].) Тот факт, что в формулировке этого вопроса величина $d+1$ не может быть заменена величиной $d$ или еще меньшей, легко следует по принципу Дирихле из рассмотрения множества вершин правильного $d$-мерного симплекса. Кроме того, аналогичное замечание может быть сделано со ссылкой на теорему Борсука о шаре: эта теорема гласит, что шар, лежаший в евклидовом пространстве размерности $d$, невозможно разрезать на $d$ "долек", диаметр которых был бы строго меншше диаметра самого шара. Доказательство этой теоремы можно найти как в оригинальной работе самого Борсука (см. [3]), так и в некоторых монографиях, посвященных сходньм вопросам комбинаторной геометрии (см., например, [4]), так что в настоящей работе мы это доказательство не обсуждаем$^{1}$; возможность же разбиения шара на $d+1$ часть меньшего диаметра очевидна. Наконец, в 1955 году Ленц доказал, что никакое $d$-мерное тело постоянной ширины (т.е., в частности, и шар тоже) не может быть разбито на $d$ частей меньшего диаметра (cM. $[6])$.

Что же нам известно о гипотезе Борсука в “малых" размерностях? Во-первых, в размерности $d=1$ гипотеза Борсука, очевидно, верна. Во-вторых, в размерности $d=2$ сам Борсук показал, что всякое ограниченное множество разбивается на три части меньшего диаметра, и, собственно, именно этот результат привел его к формулировке общей гипотезы. Доказательство Борсука опиралось на несложную геометрическую лемму о том, что всякое плоское ограниченное множество диаметра $D$ может быть заключено в правильньй шестиугольник, у которого расстояние между противоположньми сторонами также равно $D$ (см. [1], [4], [7] и [8]). Более того, из этого доказательства немедленно следовало, что каждое ограниченное двумерное множество диаметра $D$ может быть разбито на три части, диаметр каждой из которых, в свою очередь, не превосходит величины $\frac{\sqrt{3}}{2} D=0.8660 \ldots D$. Иными словами, для величины

$$
\alpha_{2}=\sup _{\Omega \subset \mathbb{R}^{2}} \inf _{\Omega_{1}, \Omega_{2}, \Omega_{3}} \max _{i=1,2,3} \operatorname{diam} \Omega_{i}
$$

Борсук получил оценку

$$
\alpha_{2} \leqslant \frac{\sqrt{3}}{2}=0.8660 \ldots
$$

\footnotetext{
${ }^{1}$ Стоит отметить, что несколько раньше Борсука - в 1930 году - тот же, по существу, результат был получен с помощью топологических методов Л. А. Люстерником и Л. Г. Шнирельманом (см. [5]).
} 
(Здесь в определении величины $\alpha_{2}$ первьй супремум берется по всем множествам $\Omega \subset$ $\mathbb{R}^{2}$ единичного диаметра, точная нижняя грань берется по всевозможньм разбиениям фиксированного множества $\Omega$ на части меньшего диаметра, а в последнем максимуме выбирается та из этих частей, у которой диаметр окажется наибольшим.) Таким образом, в размерностях 1 и 2 проблема Борсука решается достаточно просто. Однако уже в размерности $d=3$ ситуация становится несколько более сложной.

Впервые трехмерная гипотеза Борсука была доказана Перкалом и Эгглстоном, но доказательство, предложенное ими (см. [9] и [10]), было весьма длинным и неэлементарным, в отличие от только что описанного нами двумерного доказательства Борсука. Кроме того, из этого доказательства невозможно было выделить какую-либо оценку для величины

$$
\alpha_{3}=\sup _{\Omega \subset \mathbb{R}^{3}} \inf _{\Omega_{1}, \ldots, \Omega_{4}} \max _{i=1, \ldots, 4} \operatorname{diam} \Omega_{i},
$$

лучшую, чем $\alpha_{3} \leqslant 1$. (Здесь определение величины $\alpha_{3}$ полностью аналогично определению величины $\alpha_{2}$.) Результат Перкала и Эгглстона неоднократно улучшался и упрошался: так, в 1957 году Б. Грюнбаум (см. [11]) нашел элементарное доказательство трехмерной гипотезы Борсука, и доказательство это основывалось на прямом обобщении идеи Борсука. Иначе говоря, Грюнбаум также рассмотрел трехмерную “универсальную покрьшку", аналогичную шестиугольнику на плоскости, а именно, он показал (основываясь на лемме Д. Гэйла (см. [12])), что всякое множество диаметра $D$ в $\mathbb{R}^{3}$ может быть заключено в многогранник, получаемьй из правильного октаэдра, у которого расстояние между противоположными гранями в точности равно $D$, путем последовательного отсечения нескольких пирамид (подробности можно найти в оригинальной работе Грюнбаума и в [4]); многогранник же этот может быть, в свою очередь, разбит на четыре части, диаметр каждой из которых строго меньше $D$. В то же самое время, в 1957 году, А. Хепшеш (см. [13]) получил другое элементарное решение трехмерной проблемы. Это решение также основьвалось на построении некоторой универсальной покрьшки. Тем не менее у Хеппеша

$$
\alpha_{3} \leqslant \frac{\sqrt{9+4 \sqrt{3}}}{4}=0.99775 \ldots,
$$

в то время как у Грюнбаума

$$
\alpha_{3} \leqslant \frac{\sqrt{6129030-937419 \sqrt{3}}}{1518 \sqrt{2}}=0.9887 \ldots,
$$

и этот результат до недавнего времени оставался наилучшим из известных.

Однако в 1997 году В. В. Макеев доказал, что в качестве универсальной покрышки для множеств диаметра $D$ в $\mathbb{R}^{3}$ может быть взят ромбододекаэдр ${ }^{2}$ с расстоянием $D$ между противолежащими гранями, усеченньй тремя взаимно перпендикулярными плоскостями, проходящими на расстоянии $D / 2$ от центра ромбододекаэдра и отсекающими от него правильные четырехугольные пирамиды с вершинами в вершинах ромбододекаэдра (см. [14] и [15]). А Л. Евдокимов с помошью компьютера отыскал разбиение такого многогранника на четыре части, диаметр каждой из которых не превосходит $0.98 D$. Таким образом, совмешая результаты Макеева и Евдокимова, мы получаем оценку $\alpha_{3} \leqslant 0.98$, и это немного лучше, чем оценка Грюнбаума.

\footnotetext{
${ }^{2}$ Напомним, что ромбододекаэдром назьвается двенадцатигранник, двойственный к кубооктаэдру, где, в свою очередь, кубооктаэдр - это выпуклая оболочка середин ребер куба.
} 
Впрочем, вполне вероятно, что все эти оценки весьма далеки от правильной: в 1953 году Гэйл (см. [12]) высказал гипотезу, что правильной будет оценка

$$
\alpha_{3} \leqslant \frac{\sqrt{3+\sqrt{3}}}{\sqrt{6}}=0.888 \ldots
$$

Интересно, что для произвольного конечного множества точек или, что то же самое, для произвольного многогранника в $\mathbb{R}^{3}$ проблема Борсука решается намного проше: в 1956 году такой результат был получен Хеппешем и Ревесом в работе [16]. (А аналогичньй результат на плоскости вытекает из работы Эрдеша 1946 года (см. [17] и [18]).)

Наконец, в связи со всем сказанным вьше, следует отметить результат П. Кацаровой-Карановой, которая в 1967 году доказала, что всякое трехмерное множество диаметра 2 может быть покрыто четырьмя шарами радиуса 0.999983 (см. [19]). Конечно, оценка для величины $\alpha_{3}$ здесь получается крайне слабая, зато, с одной стороны, доказана гипотеза Грюнбаума о возможности покрытия всякого ограниченного трехмерного множества четырьмя шарами того же диаметра (см. [18] и [20]), а с другой стороны, предложен иной подход к доказательству гипотезы Борсука в $\mathbb{R}^{3}$.

На размерности $d=3$ заканчивается перечень "малых" размерностей, в которых к настоящему времени удалось получить окончательный результат в проблеме Борсука. Уже в четырехмерном случае неизвестно, разбивается ли всякое ограниченное множество на 5 частей меньшего диаметра. Более того, ответ на аналогичньй вопрос неизвестен в этом случае даже для многогранников.

2.2. Решение проблемы Борсука для некоторых специальных классов множеств. В 1946 году Г. Хадвигер доказал, что всякое $d$-мерное множество с гладкой гранищей может быть разбито на $d+1$ часть меньшего диаметра (см. [21]). Иначе говоря, согласно Хадвигеру, верна гипотеза Борсука, сформулированная не во всей своей полноте, а только для весьма широкого класса множеств - множеств с гладкой гранищей. Доказательство теоремы Хадвигера (а также некоторое уточнение этой теоремы, тоже принадлежашее Хадвигеру) может быть найдено в [21] и в книге [4], так что мы на нем останавливаться не будем.

Некоторьй принципиально другой, отличный от хадвигеровского, класс множеств, в рамках которого верна гипотеза Борсука, был существенно позже - в 1971 году предложен К. А. Роджерсом. Роджерс (см. [22]) доказал следуюшую теорему.

ТЕОРемА. При $d \geqslant 3$ всякое множество $\Omega \subset \mathbb{R}^{d}$ диаметра 1 , инвариантное относительно действия группь конгруэнций, оставляющей на месте правильный $d$-мерный симплекс, может быть покрыто выпуклыми замкнутылми множествами так, что число этих множеств равно $d+1$, а диаметр каждого из них не превосходит величинь

$$
\sqrt{\left(1-\frac{6-4 \sqrt{2}}{t(t+1) d}\right)}
$$

əде $t=[(d+1) / 2]$.

Сушествуют и другие общие ситуации, в рамках которых доказана гипотеза Борсука. Однако эти ситуации носят уже несколько более специальный характер. Обо всех 
этих ситуациях можно прочесть в замечательной недавно вьшедшей книге В.Г. Болтянского, Х. Мартини и П. С. Солтана (см. [23]).

Прежде чем переходить к изложению следующего раздела, автор хотел бы поблагодарить В. Г. Болтянского и Х. Мартини за копию книги [23], любезно предоставленную ими, а также за постоянную помош и поддержку. Кроме того, автор благодарит Л. Данцера за указание на статью [19] и Е. Макаи за указание на работы [14], [15] (см. раздел 2.1).

2.3. Верхние оценки на минимальное число частей меньшего диаметpa. Пусть $f$ - произвольное натуральное число такое, что всякое ограниченное точечное множество ненулевого диаметра $\Omega \subset \mathbb{R}^{d}$ может быть разбито на $f$ частей меньшего диаметра. Определим $f(d)$ как число, минимальное среди всех таких $f$. Понятно тогда, что гипотеза Борсука эквивалентна равенству $f(d)=d+1$. Конечность величины $f(d)$ доказывается очень просто: достаточно произвольное множество диаметра $D$ заключить в $d$-мерньй куб с ребром $D$, а затем разбить этот куб на столь мелкие кубики, что диаметр каждого из них уже будет меньше $D$. Это несложное обстоятельство впервые в 1955 году отметил Ленщ (см. [6]), доказавший тем самьм, что $f(d) \leqslant(\sqrt{d}+1)^{d}$. Сперва этот результат слегка улучшил К. Борсук (см. [24]): в 1978 году он показал, что имеет место оценка

$$
f(d) \leqslant m_{1} \times \cdots \times m_{d}, \text { если } \sum_{i=1}^{d} m_{i}^{-2}<1 .
$$

(Нетрудно видеть, что такая оценка действительно является улучшением результата Ленца, поскольку путем элементарных преобразований ее можно привести к виду $f(d) \leqslant q_{d}^{d-s_{d}}\left(q_{d}-1\right)^{s_{d}}$, где $q_{d}$ - наименьшее целое число, большее, чем $\sqrt{d}$, а $s_{d}$ - наибольшее целое число, меньшее, чем $\left(q_{d}^{d}-d\right)\left(q_{d}-1\right)^{2}\left(2 q_{d}-1\right)^{-1}$.) Позже, в 1982 году, М. Лассак (см. [25]) сумел существенно улучшить эту оценку, заметив сначала, что уже из классической теоремы Юнга (см. [26]), говоряшей о сушествовании шара радиуса $\sqrt{d /(2 d+2)}$, описанного вокруг произвольного $d$-мерного множества диаметра 1 , вытекает неравенство ${ }^{3} f(d) \leqslant 2^{d}$, а затем доказав, что, более того, $f(d) \leqslant 2^{d-1}+1$. Точнее, Лассак доказал следующую теорему.

Tеорема. Всякое множество диаметра 1 в $\mathbb{R}^{d}$ может быть разбито на $2^{d-1}+1$ часть так, что диаметр каждой из этих частей будет не больше величинь

$$
\sqrt{\frac{4 d^{2}+\sqrt{8 d^{2}+1}-1}{4 d^{2}+4 d}}
$$

Однако даже такой результат крайне далек от ожидаемого (разумеется, если не брать в расчет случаи $d=1$ и $d=2$ ). Полезно отметить, кстати, что доказательство теоремы Лассака основано на построении следующей простой универсальной покрьшки: пусть $\mathscr{B}_{1} \subset \mathbb{R}^{d}$ - произвольный $d$-мерный шар радиуса $\sqrt{d /(2 d+2)}$, $\mathbf{x} \in \partial \mathscr{B}_{1}$ - произвольная точка, лежашая на границе этого шара, а $\mathscr{B}_{2}$ - шар радиуса 1 с центром в точке $\mathbf{x}$; тогда в качестве универсальной покрышки берется

\footnotetext{
${ }^{3}$ Отметим, что аналогичное замечание еще раньше - в 1974 году - сделал Р. Кнаст в своей работе [27].
} 
множество $\Omega=\mathscr{B}_{1} \cap \mathscr{B}_{2}$ (нетрудно проверить, что всякое множество диаметра 1 в $\mathbb{R}^{d}$ действительно покрьвается некоторой жесткой копией, т.е. образом под действием движения, множества $\Omega$ - см. [25] и [26]).

Наконец, в 1988 году О. Шрамм сформулировал следующий, не улучшенный до сих пор результат (см. [28]):

$$
f(d)<5 d \sqrt{d}(4+\log d)\left(\frac{3}{2}\right)^{d / 2}=(1.224 \ldots+o(1))^{d} .
$$

Таким образом, никаких оценок сверху на величину $f(d)$, лучших, чем экспоненциальные, никто до сих пор не знает. Результат же Шрамма получается в качестве следствия из другой теоремы Шрамма, даюшей верхнюю оценку для числа освещения произвольного множества постоянной ширины. Поясним сказанное несколько подробнее.

Говорят, что направление $\mathbf{u} \in S^{d-1}$, где $S^{d-1}-(d-1)$-мерная сфера в $\mathbb{R}^{d}$, освещает вьпуклое тело (компактное вьпуклое множество с непустой внутренностью) $\Omega$ в точке $\mathbf{x}$, лежащей на границе этого тела, если при некотором положительном $t$ точка $\mathbf{x}+t \mathbf{u}$ является внутренней точкой множества $\Omega$. Говорят также, что направления $\mathbf{u}_{1}, \ldots, \mathbf{u}_{m} \in S^{d-1}$ освещают тело $\Omega$, если в каждой точке своей гранищы это тело освешено хотя бы одним из этих направлений. Наконец, через $I(\Omega)$ обозначается минимальное число таких направлений для заданного множества $\Omega$. Величину $I(\Omega)$ принято называть числом освешения тела $\Omega$.

Обозначим теперь через $H=H(\Omega)$ минимальное число такое, что множество $\Omega$ представляется в виде:

$$
\Omega=\Omega_{1} \cup \cdots \cup \Omega_{H},
$$

где каждое множество $\Omega_{i}$ гомотетично исходному множеству $\Omega$ с коэффициентом гомотетии, меньшим единицы. Тогда для вьпуклого ограниченного множества $\Omega$ имеет место теорема В. Г. Болтянского о том, что $I(\Omega)=H(\Omega)$ (см. [29], [4] и [23]).

Шрамм же доказал, что если $\Omega$ - множество постоянной ширины, то

$$
I(\Omega)<5 d \sqrt{d}(4+\log d)(1.5)^{d / 2} .
$$

Но тогда по теореме Болтянского то же неравенство выполнено и для $H(\Omega)$. С другой стороны, известно (см., скажем, [30]), что всякое множество $S \subset \mathbb{R}^{d}$ диаметра $D$ может быть заключено в множество $\Omega$ постоянной ширины и того же диаметра. Стало быть, $H(S) \leqslant H(\Omega)$ и упомянутая выше оценка для величины $f(d)$ в задаче Борсука действительно имеет место.

В этом разделе следует также упомянуть теорему Ж. Бургейна и И. Линденштраусса (см. [31]) 1991 года, которая слегка уточняет результат Шрамма: всякое множество диаметра 1 в $\mathbb{R}^{d}$ может быть покрыто $(\sqrt{3 / 2}+\varepsilon)^{d}$ множествами, диаметр каждого из которых не больше величины $(1-\varepsilon / 2)$, при условии, что $d \geqslant d(\varepsilon)$. Эта теорема основана на применении следующего предложения.

ПРЕДЛОЖЕНИЕ. Пусть $c_{d}$ - это минимальное число такое, что всякое множество в $\mathbb{R}^{d}$ диаметра 1 может быть покрыто $c_{d}$ шарами того же диаметра. Тогда для любого $\varepsilon>0$ и для $d>d(\varepsilon)$

$$
(\sqrt{9 / 8}-\varepsilon)^{d} \leqslant c_{d} \leqslant(\sqrt{3 / 2}+\varepsilon)^{d} .
$$


Иными словами, здесь мы имеем дело с подходом, аналогичным тому, с которым мы уже встречались при обсуждении работы Кацаровой-Карановой (см. [18], [19] и [20]). Заметим, кстати, что еще в 1965 году Л. Данщер доказал, что $c_{d} \geqslant(1.003)^{d}$ (см. [32]). Более того, из одной работы Роджерса 1963 года (см. [33]) нетрудно вьвести оценку $c_{d} \leqslant(\sqrt{2}+o(1))^{d}$. Однако явное указание на это обстоятельство впервые появилось только лишш в работе Шрамма 1987 года (см. [34]). ${ }^{4}$

Прежде чем окончить настояший раздел, представляется интересным проследить, как описанные в этом разделе оценки работают в размерности $d=4$. Действительно, из работы [6] следует, по-видимому, очень грубая оценка $f(4) \leqslant 81$. Результат работы [24] позволяет доказать гораздо более точное неравенство $f(4) \leqslant 24$. Наконец, основная теорема Лассака из работы [25] дает оценку $f(4) \leqslant 9$. К сожалению, из работ [28], [31], [33] и [34] невозможно вьвести никаких дальнейших улучшений: с ростом размерности они сушественно усиливают неравенство Лассака; однако в малых размерностях ситуация оказывается полностью противоположной.

2.4. Контрпримеры к гипотезе Борсука и нижние оценки на минимальное число частей меньшего диаметра. Из предыдущих разделов видно, что различных подходов к решению проблемы Борсука существует достаточно много, и тем не менее ни один из них не дает желаемого результата. Еще Роджерс в своей статье 1971 года о разбиении симметричных множеств (см. [22]) писал, что получил основной результат этой статьи, безуспешно пытаясь опровергнуть гипотезу Борсука. А 10 лет спустя П. Эрдеш высказал предположение о возможности построения контрпримера к гипотезе Борсука, основанного на изучении комбинаторики некоторых конечных точечных конфигураций в $\mathbb{R}^{d}$ (см. [35]). Одновременно аналогичное предположение высказал и Д. Ларман (см. [36] и [37]).

Наконец, в 1993 году гипотеза Борсука, действительно, была опровергнута: Й. Кан и Г. Калаи, основьваясь на результатах работы П. Франкла и Р. Вилсона 1981 года (см. [38]), построили контрпример к гипотезе во всех размерностях $d \geqslant 2015$ (см. [39]). Более того, они показали, что $f(d) \geqslant(1.203 \ldots+o(1))^{\sqrt{d}}$, и в свете этой нижней оценки результаты Шрамма и Бургейна-Линденштраусса представляются уже не столь разочаровьвающими. В дальнейшем были предприняты достаточно многочисленные попытки понижения размерности контрпримера и, одновременно, усиления нижней оценки на величину $f(d)$. Так, в 1994 году А. Нилли предложил некоторую модификацию метода Кана и Калаи и в результате нашел отрицательное решение проблемы Борсука при $d \geqslant 946$ (см. [40]). Кроме того, работа Нилли была краткой и, в отличие от работы Кана и Калаи, полностью замкнутой в себе. Далее, в 1997 году Й. Грэй и Б. Вайсбах построили, за счет небольшого уточнения метода Нилли, контрпримеры для всех $d \geqslant 903$ (см. [41]). В том же году автор (см. [42]) обнаружил иную модификацию подхода Нилли, которая позволила ему опровергнуть гипотезу Борсука уже при $d=561$. Наконец, в 2000 году Б. Вайсбах слегка видоизменил конструкцию из [42], сумев тем самым уменьшить размерность контрпримера еще на единицу (см. [43]). Следует еще добавить, что в 1999 году в своей работе [44] автор доказал также несправедливость гипотезы Борсука при всех $d \geqslant 561$. Аналогичньй результат был в том же году независимо получен Д. Гуревичем и А. Гайфуллиньм (см. [45]).

О величине $f(d)$ известно гораздо меньше: единственный результат, улучшающий оценку из работы [39], был получен в 1999 году автором, которому с помощью еще

\footnotetext{
${ }^{4}$ Впрочем, схожие замечания могут быть найдены и в книге [20].
} 
одного обобщения метода Нилли удалось показать, что $f(d) \geqslant(2 / \sqrt{3}+o(1))^{\sqrt{2} \sqrt{d}}=$ $(1.225 \ldots+o(1))^{\sqrt{d}}($ см. [44]).

Отметим, что метод, позволивший Кану и Калаи построить многомерньй контрпример к гипотезе Борсука, настолько прост и изящен, что одна из его модификаций даже попала в книгу М. Айгнера и Г. М. Циглера "Proofs from the BOOK" ("Доказательства из КНИГИ") (см. [46]), в которой собраны самые тонкие и изяшные - “совершенные" - доказательства, т.е. именно те доказательства, что содержатся по мнению Эрдеша у Бога в особой КНИГЕ.

К изложению метода Кана и Калаи, его модификаций и их применению мы вернемся в $\S 4$, где будут даны доказательства многих результатов, представленных в этом и следуюшем параграфах, а также сформулирован общий метод, о котором шла речь во введении. В третьем же параграфе мы будем обсуждать хроматические числа различных метрических пространств.

В заключение скажем, что с некоторьми другими аспектами проблемы Борсука, а также с многочисленными смежными результатами и задачами комбинаторной геометрии можно ознакомиться, во-первых, по уже не раз цитированной книге Болтянского и Гохберга (см. [4]), во-вторых, по книге Болтянского, Мартини и Солтана (см. [23]) и, кроме того, по работам [47], [48], [49] и [50; с. 123-125].

\section{§ 3. Хроматические числа некоторых метрических пространств}

В своей знаменитой неопубликованной работе 1976 года (см. [51] и [52]) М. Бенда и М. Перлес дали следующее общее определение хроматического числа.

ОПРЕДЕЛЕНИЕ. Пусть $(X, r)$ - некоторое метрическое пространство, а $a$ - произвольное неотрицательное вешественное число. Рассмотрим граф $G_{a}$, множество вершин которого совпадает со всем пространством $X$, а множество ребер состоит из всех возможных пар различных точек $x, y \in X$ таких, что $r(x, y)=a$. Тогда хроматическим числом метрического пространства $X$ с запрещенным (или критическим) расстоянием $a$ назьвается величина $\chi(X, a)=\chi\left(G_{a}\right)$ (здесь $\chi\left(G_{a}\right)$ - хроматическое число графа $G_{a}$, определяемое стандартно, см., например, [53]).

Иными словами, хроматическое число метрического пространства - это минимальное число цветов, в которые можно раскрасить точки пространства так, что точки, отстояшие друг от друга на расстояние $a$, окажутся раскрашенными в различные цвета. В случае, когда в роли метрического пространства $(X, r)$ выступает пространство $\mathbb{R}^{d}$, снабженное стандартной евклидовой метрикой, мы имеем дело с классической задачей о нахождении хроматического числа евклидова пространства, впервые сформулированной Нелсоном и Исбелом, а также, независимо, Эрдешем и Хадвигером. В настоящей работе мы изучим, главным образом, те вопросы, которые связаны с получением оценок для величин $\chi\left(\mathbb{R}^{d}\right)=\chi\left(\mathbb{R}^{d}, 1\right)$ и $\chi\left(\mathbb{Q}^{d}\right)=\chi\left(\mathbb{Q}^{d}, 1\right)$, где $\mathbb{Q}^{d} \subset \mathbb{R}^{d}$ - множество всех точек вешественного пространства, имеюших рациональные координаты. Кроме того, мы рассмотрим некоторые новые обобщения понятия хроматического числа пространства.

3.1. Некоторые предварительные замечания. Прежде всего следует отметить, что конечность величины $\chi\left(\mathbb{R}^{d}\right)$, а стало быть, и величины $\chi\left(\mathbb{Q}^{d}\right)$, может быть доказана столь же легко, как это было сделано для величины $f(d)$ в $\S 2$ : действительно, достаточно все евклидово пространство размерности $d$ замостить кубами с ребром 
длины 2 и вершинами в точках решетки $\mathbb{Z}^{d}$, затем каждьй из этих кубов разбить на одинаковые мелкие кубики, диагонали которых строго меньше единищы, далее, каждому мелкому кубику в каком-нибудь из больших кубов придать свой, отдельньй, цвет и, наконец, перенести эту раскраску параллельньми переносами на все остальные кубы. Ясно, что таким образом мы получим верхнюю оценку хроматического числа вида $\chi\left(\mathbb{Q}^{d}\right) \leqslant \chi\left(\mathbb{R}^{d}\right) \leqslant d^{c d}$ с некоторой константой $c>0$.

Также может возникнуть следующий вопрос: верно ли, что хроматическое число пространства $\mathbb{R}^{d}$ совпадает с хроматическим числом некоторого конечного подграфа в графе, использованном для определения величины $\chi\left(\mathbb{R}^{d}\right)$ ? Иначе говоря, верно ли, что $\max \chi(G)=\chi\left(\mathbb{R}^{d}\right)$, если максимум берется по всем конечным графам $G=(\mathscr{V}, \mathscr{E})$ таким, что $\mathscr{V} \subset \mathbb{R}^{d}$, а $\mathscr{E}=\{x, y \in \mathscr{V}: d(x, y)=1\}$ (здесь, как и во введении, $d(x, y)$ - евклидова метрика)? Ответ на этот вопрос выглядит так: если мы принимаем аксиому выбора, то по теореме Эрдеша-де Брёйна (см. [54] и [55]) $\max \chi(G)=\chi\left(\mathbb{R}^{d}\right)$ (поскольку мы только что доказали, что $\chi\left(\mathbb{R}^{d}\right)<\infty$ ) и вопрос тем самьм решается положительно; однако в противном случае общего результата нет, а есть лишь примеры, показывающие, что, вообще говоря, ответ может получиться и отрицательным. Так, в 1984 году Л.А. Секели предложил следующую конструкцию (см. [56]): рассмотрим единичную окружность $S^{1} \subset \mathbb{R}^{2}$; пусть $\alpha<\pi$ - такое положительное вещественное число, что $\alpha / \pi \notin \mathbb{Q}$; определим граф $G_{\alpha}=\left(\mathscr{V}_{\alpha}, \mathscr{E}_{\alpha}\right)$ следующим образом: множество вершин $\mathscr{V}_{\alpha}$ этого графа совпадает со всей окружностью $S^{1}$, а ребрами соединены те и только те вершины, для которых длина минимальной соединяющей дуги равна $\alpha$. Допустим, все множества измеримы по Лебегу (если бы мы принимали аксиому выбора, это заведомо было бы не так: хорошо известньй пример множества, не измеримого по Лебегу, легко строится с помошью аксиомы выбора). Тогда $\chi\left(G_{\alpha}\right)=3$, в то время как $\max \chi(G)=2$ (здесь максимум берется по всевозможньм конечным подграфам в $\left.G_{\alpha}\right)$. Заметим, что наше предположение об измеримости всех множеств совместимо с аксиоматикой Цермело-Френкеля (ZF) и несовместимо с аксиоматикой ZFC (см., скажем, [57]).

3.2. Оценки хроматических чисел в "малых" размерностях. 1. Понятно, что при $d=1 \quad \chi\left(\mathbb{Q}^{d}\right)=\chi\left(\mathbb{R}^{d}\right)=2$.

2. Однако уже на плоскости ситуация кардинально меняется. Если про хроматическое число рационального пространства известно (см. [58]), что оно в точности равно двум (как и на прямой), то для величины $\chi\left(\mathbb{R}^{2}\right)$ имеются лишь оценки:

$$
4 \leqslant \chi\left(\mathbb{R}^{2}\right) \leqslant 7 .
$$

Обе эти оценки были доказаны в 1961 году - нижняя Л. Мозером и В. Мозером, а верхняя Хадвигером (см. [59] и [60]), - и никаких принципиальных улучшений с тех пор найдено не было.

3. При $d=3$ известно, что:

$$
\chi\left(\mathbb{Q}^{d}\right)=2, \quad 5 \leqslant \chi\left(\mathbb{R}^{d}\right) \leqslant 18 .
$$

Здесь результат для рационального случая был получен Бендой и Перлесом, нижняя оценка для вешественного случая была в 1970 году доказана Д. Е. Райским (см. [61]), а верхняя - в 1997 году - Кулсоном (см. [62]). Более того, совсем недавно Кулсон объявил, что может доказать неравенство $\chi\left(\mathbb{R}^{3}\right) \leqslant 15$, но этот результат пока не опубликован. 
4. В четырехмерном пространстве опять-таки известно точное значение величины $\chi\left(\mathbb{Q}^{4}\right)$. Оно равно четырем, и результат этот восходит к работе Бенды и Перлеса (см. [51]). Для вещественного же случая имеется только нижняя оценка $\chi\left(\mathbb{R}^{4}\right) \geqslant 6$ (см. [61]).

5. При $d=5$ К.Б. Чилакамарри доказал в 1990 году, что $\chi\left(\mathbb{Q}^{d}\right) \geqslant 6$ (cм. [63]). Известно также (см. [64]), что $\chi\left(\mathbb{Q}^{d}\right) \leqslant 8$. Это позволило Чилакамарри высказать в 1993 году гипотезу о том, что $\chi\left(\mathbb{Q}^{5}\right)=8$. Совсем недавно М. Манн объявил, что сумел с помошюю компютера доказать оценку $\chi\left(\mathbb{Q}^{5}\right) \geqslant 7$. Для вешественного же случая здесь остается наилучшей оценка Д. Лармана и К. А. Роджерса: $\chi\left(\mathbb{R}^{5}\right) \geqslant 8$. Эта оценка была получена в 1972 году (см. [65]).

6. В случае, когда $d=6, \breve{И}$. Закс сначала получил оценку $\chi\left(\mathbb{Q}^{d}\right) \geqslant 6($ см. [66]), а затем улучшил ее, показав, что $\chi\left(\mathbb{Q}^{d}\right) \geqslant 7$ (см. [67]). Так же, как и в пятимерном случае, для $\mathbb{R}^{d}$ здесь “рекорд" принадлежит Ларману и Роджерсу: $\chi\left(\mathbb{R}^{d}\right) \geqslant 10$ (см. [65]).

7. При $d=7$ все наилучшие результаты для рационального случая так же, как и при $d=6$, принадлежат Заксу: в [66] он получил оценку $\chi\left(\mathbb{Q}^{d}\right) \geqslant 7$, а в [67] уточнил ее $-\chi\left(\mathbb{Q}^{d}\right) \geqslant 9$. Для вешественного случая долгое время наилучшей оставалась оценка Лармана и Роджерса 1972 года (см. [65]), однако в самое последнее время автор улучшил эту оценку, доказав, что $\chi\left(\mathbb{R}^{d}\right) \geqslant 15$.

8. При $d=8$ имеют место неравенства $\chi\left(\mathbb{Q}^{d}\right) \geqslant 10$ (см. [67], а также [66], где доказано, что $\left.\chi\left(\mathbb{Q}^{d}\right) \geqslant 9\right)$ и $\chi\left(\mathbb{R}^{d}\right) \geqslant 16($ см. $[65])$.

9. В размерности $d=9$ наилучшим для рационального случая является недавний результат автора: $\chi\left(\mathbb{Q}^{d}\right) \geqslant 12$. Для вещественного же случая лучшая оценка принадлежит Ларману и Роджерсу: $\chi\left(\mathbb{R}^{d}\right) \geqslant 16$ (см. [65]). На этой размерности заканчивается перечень размерностей, в которых когда-либо специально рассматривался случай рационального пространства.

10 . В случае, когда $10 \leqslant d \leqslant 14$ и рассматриваемое пространство вешественное, до последнего времени самыми точными были оценки Лармана и Роджерса, основанные на некоторой неопубликованной лемме П. Эрдеша и В. Шош. Эти оценки можно найти в работе [65] и в статье Секели [68], так что здесь мы их не приводим. Тем не менее в размерности $d=11$ автору удалось слегка улучшить упомянутые результаты и доказать, таким образом, неравенство $\chi\left(\mathbb{R}^{d}\right) \geqslant 20$.

Весьма подробный обзор и доказательства большинства этих результатов можно также найти: для вещественного случая - в работе Л. Секели (см. [68] и, кроме того, [69]); для рационального случая - в работе К. Чилакамарри (см. [64]). Поэтому в настоящей статье мы эти результаты детально не обсуждаем. Недавние же результаты автора, перечисленные в этом разделе, будут доказаны в $\S 4$.

Прежде чем приступить к дальнейшему изложению, автор хотел бы поблагодарить Л. Секели за любезно предоставленный обзор [68], а также за постоянную помошь и поддержку. Кроме того, автор благодарит Й. Закса, П. Д. Джонсона и Н. Алона за помощь, без которой данная глава вряд ли могла быть написана.

3.3. Оценки хроматических чисел с ростом размерности и реализация всех расстояний при разбиении пространства $\mathbb{R}^{d}$ на некоторое число частей. В этом разделе мы дадим обзор результатов, связанных с получением асимптотических оценок для величин $\chi\left(\mathbb{R}^{d}\right)$ и $\chi\left(\mathbb{Q}^{d}\right)$. Также мы дадим некоторые ссылки, касающиеся результата Хадвигера, упомянутого нами во введении. Напомним, что Хадвигер доказал в 1944 году (см. [2]) следующую теорему. 
Теорема 1. Пусть $\mathbb{R}^{d}$ покрыто $d+1$ замкнутым множеством. Тогда найдется такое мноэсество, принадлежащее этому покрытию, что в нем все неотрицательные вещественные числа реализуются как попарные расстояния межуду его точками.

По-видимому, первым, кто получил нетривиальную нижнюю оценку для хроматического числа вещественного пространства в произвольной размерности $d$, был Д.Е. Райский, доказавший, что $\chi\left(\mathbb{R}^{d}\right) \geqslant d+2$ (см. [61]). Результат этот Райский получил путем прямого обобшения идей Л. Мозера и В. Мозера (см. [59]). Кроме того, Райский усилил теорему Хадвигера о реализации всех расстояний, показав, что условие замкнутости множеств из формулировки этой теоремы может быть удалено. Заметим, что этот же результат был получен независимо Вудалом (см. [58]). Далее, в 1972 году Д. Ларман и К.А. Роджерс (см. [65]) добились существенного продвижения в вопросе получения нижних оценок для величины $\chi\left(\mathbb{R}^{d}\right)$. Сперва им удалось установить оценку $\chi\left(\mathbb{R}^{d}\right) \geqslant c_{1} \frac{d^{2}}{(\log d)^{3}}$, где $c_{1}>0$ - абсолютная постоянная, а затем получить (с использованием неопубликованной леммы Эрдеша и Шош) неравенство $\chi\left(\mathbb{R}^{d}\right) \geqslant c_{2} d^{2}$ (здесь $c_{2}$ - также абсолютная постоянная). Одновременно ими была доказана следующая теорема.

Tеорема 2. Пусть $N_{d} \sim \frac{d^{2}}{\left(\log _{2} d\right)^{3}}$. Тогда:

(а) если измеримое множсство в $\mathbb{R}^{d}$ имеет верхнюю плотность, превышающую $1 / N_{d}$, то в әтом множестве реализуются все расстояния;

(b) если все $\mathbb{R}^{d}$ покрыто меньше чем $N_{d}$ множсествами, то хотя бы в одном из этих множеств реализуются все расстояния.

В дальнейшем результаты Лармана и Роджерса неоднократно улучшались: так, в 1978 году сам Ларман вернулся к изучению вопроса и показал, что $\chi\left(\mathbb{R}^{d}\right) \geqslant(d-1)(d-2)(d-3) / 178200$ (см. [70]); а в 1980 году П. Франкл получил неравенство $\chi\left(\mathbb{R}^{d}\right) \geqslant n^{t}$, где $t$ любое, а $n>n(t)$ (см. [71]). Прорыв был совершен в 1981 году в замечательной работе П. Франкла и Р. Вилсона, в той самой работе, которую мы уже однажды цитировали в связи с проблемой Борсука и контрпримером Кана и Калаи (см. [38]). В этой работе авторам удалось доказать гипотезу Эрдеша об экспоненщиальности роста величины $\chi\left(\mathbb{R}^{d}\right)$. Точнее, они оценили снизу хроматическое число экспонентой $(1.207 \ldots+o(1))^{d}$, и этого уже было достаточно для доказательства гипотезы Эрдеша, поскольку еше в 1972 году Ларман и Роджерс, используя результаты Батлера и метод Эрдеша и Роджерса, доказали, что $\chi\left(\mathbb{R}^{d}\right) \leqslant(3+o(1))^{d}$ (см. [65], [72] и [73]). Более того, Франкл и Вилсон показали, что в теореме Лармана и Роджерса о реализации всех расстояний величина $N_{d}$ также может быть взята равной $(1.207 \ldots+o(1))^{d}$.

Наконец, в 2000 году автор доказал следующую теорему (см. [74]).

ТЕОрема 3. Положим

$$
\begin{array}{ll}
A_{1}=\frac{x+2 y}{2}, & A_{2}=\sqrt{-3 A_{1}^{2}+6 A_{1}+1}, \\
A_{3}=\frac{1}{4}\left(1+\frac{A_{1}-1}{A_{2}}\right), & A_{4}=\frac{1}{6}\left(1+3 A_{1}-A_{2}\right)
\end{array}
$$


(здесь х и - независимые вещественные переменные). Определим, далее, величины $x_{0}, y_{0}$ как такие корни системы нелинейных уравнений (*), (**)

$$
\begin{gathered}
2 A_{3} \log A_{4}+\left(1-4 A_{3}\right) \log \left(A_{1}-2 A_{4}\right) \\
+\left(2 A_{3}-1\right) \log \left(1-A_{1}+A_{4}\right)-\log y+\log (x-y)=0 \\
\frac{(1-x)^{2} y}{(x-y)^{3}}=1
\end{gathered}
$$

что $x_{0}=0.36063 \ldots$, a $y_{0}=0.063907 \ldots$ Наконец, зададим величину $\gamma$ равенством

$$
\begin{gathered}
\gamma=\left(A_{4}^{0}\right)^{A_{4}^{0}}\left(A_{1}^{0}-2 A_{4}^{0}\right)^{A_{1}^{0}-2 A_{4}^{0}}\left(1-A_{1}^{0}+A_{4}^{0}\right)^{1-A_{1}^{0}+A_{4}^{0}} \\
\times\left(1-x_{0}\right)^{x_{0}-1}\left(x_{0}-y_{0}\right)^{y_{0}-x_{0}} y_{0}^{-y_{0}}
\end{gathered}
$$

где $A_{i}^{0}=A_{i}\left(x_{0}, y_{0}\right)$. Тогда $\chi\left(\mathbb{R}^{d}\right) \geqslant(\gamma+o(1))^{d}=(1.239 \ldots+o(1))^{d}$.

Таким образом, сформулированная теорема слегка уточняет оценку из [38].

С методами, с помошью которых были получены результаты работ [58], [61], [65], [70] и [71], можно ознакомиться по обзору [68], так что мы на них не останавливаемся. К результатам же работ [38] и [74] мы вернемся в $\S 4$.

И несколько слов о рациональном случае. Здесь нет никаких верхних оценок, лучших, чем те, что были получены в 1972 году Ларманом и Роджерсом для случая $\mathbb{R}^{d}$. Наилучшие же нижние оценки до последнего времени принадлежали Франклу и Вилсону, из работы которых (см. [38] и [75]) непосредственно следует, что $\chi\left(\mathbb{Q}^{d}\right) \geqslant$ $(1.139 \ldots+o(1))^{d}$. Однако в самое последнее время автор слегка улучшил эти оценки в следуюшей теореме.

ТеОРема 4. Пусть $l$ - некоторая константа, лежащая на полуинтервале $\left(\frac{1}{8}, \frac{1}{2}\right]$. Определим $y_{l}$ как корень уравнения $y(1-2 l+2 y)^{2}=(2 l-3 y)^{3}, 0<y_{l}<l / 2$ (нетрудно показать, что тем самым величина $y_{l}$ определена однозначно). Положим далее,

$$
\begin{gathered}
z_{l}=\frac{3 l+1-\sqrt{-3 l^{2}+6 l+1}}{6}, \\
\gamma_{l}=\frac{z_{l}^{z_{l}}\left(l-2 z_{l}\right)^{l-2 z_{l}}\left(1-l+z_{l}\right)^{1-l+z_{l}}}{\left(1-2 l+2 y_{l}\right)^{1-2 l+2 y_{l}} y_{l}^{y_{l}}\left(2 l-3 y_{l}\right)^{2 l-3 y_{l}}}, \quad \gamma=\min _{\frac{1}{8}<l \leqslant \frac{1}{2}} \gamma_{l} .
\end{gathered}
$$

Tогда $\chi\left(\mathbb{Q}^{d}\right) \geqslant(\gamma+o(1))^{d}$.

ЗАмечАниЕ. Можно показать, что, на самом деле, минимум, фигурирующий в формулировке теоремы 4 , достигается при $l=\frac{1}{2}$, так что $\chi\left(\mathbb{Q}^{d}\right) \geqslant\left(\gamma_{1 / 2}+o(1)\right)^{d}=$ $(1.173 \ldots+o(1))^{d}$ и оценка в теореме 4 действительно слегка улучшает оценку ла-Вилсона.

К изложению этих результатов мы также вернемся в $§ 4$.

Здесь автор хотел бы поблагодарить $\Phi$. Лазебника за помощь в отыскании копии книги Бабаи и Франкла [75]. 
3.4. Одно обобщение понятия хроматического числа пространства. В этом разделе мы рассмотрим некоторое естественное обобщение понятия хроматического числа. Следуя работе Бенды и Перлеса (см. [51]), дадим следующее определение.

ОПРЕДЕЛЕНИЕ. Пусть $(X, r)$ - некоторое метрическое пространство, а $a_{1}, \ldots, a_{k}-$ произвольный набор из $k$ неотрищательных вещественных чисел. Рассмотрим граф $G_{a_{1}, \ldots, a_{k}}$, множество вершин которого совпадает со всем пространством $X$, а множество ребер состоит из всех возможных пар различных точек $x, y \in X$ таких, что $r(x, y) \in\left\{a_{1}, \ldots, a_{k}\right\}$. Тогда хроматическим числом метрического пространства $X$ с запрешенным (или критическим) набором расстояний $a_{1}, \ldots, a_{k}$ назьвается величина $\chi\left(X, a_{1}, \ldots, a_{k}\right)=\chi\left(G_{a_{1}, \ldots, a_{k}}\right)$.

Для такого хроматического числа в случае, когда роль метрического пространства играет евклидово пространство $\mathbb{R}^{d}$, автор доказал следующую теорему.

ТЕОРемА. Пусть фиксирована некоторая размерность d и некоторое натуральное число $k$. Тогда найдется такой набор запрещенньх расстояний $a_{1}, \ldots, a_{k}$, что $\chi\left(\mathbb{R}^{d}, a_{1}, \ldots, a_{k}\right) \geqslant\left(c_{1} k\right)^{c_{2} d}$, где $c_{1}>0, c_{2}>0$ - некоторье абсолютние постоянные. Более того, при $k=2$ эту оценку можно уточнить: положим

$$
\begin{array}{ll}
A_{1}=\frac{x+2 y}{3}, & A_{2}=\sqrt{-3 A_{1}^{2}+6 A_{1}+1}, \\
A_{3}=\frac{1}{6}\left(1+\frac{A_{1}-1}{A_{2}}\right), & A_{4}=\frac{1}{6}\left(1+3 A_{1}-A_{2}\right)
\end{array}
$$

(здесь $x$ и $у$ - независимые вещественные переменные). Обозначим через $A_{5}$ выражение

$$
A_{5}=A_{3} \log A_{4}+\left(\frac{1}{3}-2 A_{3}\right) \log \left(A_{1}-2 A_{4}\right)+\left(A_{3}-\frac{1}{3}\right) \log \left(1-A_{1}+A_{4}\right) .
$$

Определим, далее, величины $x_{0}, y_{0}$ как такие корни системы нелинейных уравнений (i), (ii)

$$
\begin{gathered}
2 A_{5}-\log y+\log (x-y)=0, \\
A_{5}+\log (1-x)-\log (x-y)=0,
\end{gathered}
$$

что $x_{0}=0.4355 \ldots$, а $y_{0}=0.1091 \ldots$. Наконец, зададим величину $\gamma$ равенством (***) из формулировки теоремы 3 в разделе 3.3. Тогда найдется два вещественных числа $a_{1}>0, a_{2}>0$ таких, что

$$
\chi\left(\mathbb{R}^{d}, a_{1}, a_{2}\right) \geqslant(\gamma+o(1))^{d}=(1.439 \ldots+o(1))^{d} .
$$

Эта теорема будет доказана в четвертом параграфее с использованием общего метода, о котором шла речь во введении. В действительности, аналогичная теорема может быть сформулирована и для $\chi\left(\mathbb{Q}^{d}, a_{1}, \ldots, a_{k}\right)$. Однако формулировка и доказательство этой теоремы столь близки к формулировке и доказательству “вешественного" результата, что в настоящей работе мы их не приводим. 
ЗАмечАниЕ. Нетрудно показать, что из результатов Лармана и Роджерса [65] немедленно следует оценка $\chi\left(\mathbb{R}^{d}, a_{1}, \ldots, a_{k}\right) \leqslant c^{k d}$, где $c$-некоторая абсолютная постоянная, а величины $a_{1}>0, \ldots, a_{k}>0$ выбраны произвольным образом. Тем самым нижняя оценка, полученная в теореме, "хороша" (т.е. близка в некотором смысле к оценке Лармана-Роджерса) лишв в тех случаях, когда $k$ фиксировано, а размерность $d$ неограниченно возрастает. В противном случае между верхней и нижней оценками возникает очень большой зазор, и устранение этого зазора (или, по крайней мере, сушественное его уменьшение) представляется весьма интересной задачей.

Автор благодарит С. В. Конягина за интерес, проявленньй им в отношении результатов данного параграфа, и за ряд полезных замечаний.

В заключение заметим, что с многочисленными аспектами проблематики, рассмотренной в $\S 2$, можно также ознакомиться по книге С. Вагона и В. Кли (см. [76]). Кроме того, множество интересных ссылок и результатов может быть найдено в работах [64], $[68],[77],[78]$ и $[79]$.

\section{§4. Доказательства некоторых результатов}

В этом параграфе мы дадим доказательства некоторых теорем и результатов, сформулированных в $\S 2$ и $\S 3$. В частности, мы изложим здесь общий метод построения контрпримеров к гипотезе Борсука и получения нижних оценок как минимального числа частей меньшего диаметра, так и хроматических чисел вещественного и рационального пространств. Метод этот и результаты, связанные с ним, мы изложим в разделе 4.2. В разделе же 4.1 мы дадим доказательства недавних результатов автора, касающихся хроматических чисел в малых размерностях: $\chi\left(\mathbb{Q}^{9}\right) \geqslant 12, \chi\left(\mathbb{R}^{7}\right) \geqslant 15$ и $\chi\left(\mathbb{R}^{11}\right) \geqslant 20$.

4.1. Оценки величин $\chi\left(\mathbb{Q}^{d}\right)$ и $\chi\left(\mathbb{R}^{d}\right)$ при малых значениях $d$. 1. Начнем с рассмотрения рационального случая и докажем, что, действительно, $\chi\left(\mathbb{Q}^{9}\right) \geqslant 12$. Для этого определим в пространстве $\mathbb{Q}^{9}$ два семейства векторов $-\mathscr{F}_{1,1,1}$ и $\mathscr{F}_{1}-$ следующим образом:

$$
\begin{aligned}
\mathscr{F}_{1,1,1} & =\left\{F=\left(f_{1}, \ldots, f_{9}\right): f_{i} \in\{0,1\} \forall i=1, \ldots, 9 ; f_{1}+\cdots+f_{9}=3\right\}, \\
\mathscr{F}_{1} & =\left\{F=\left(f_{1}, \ldots, f_{9}\right): f_{i} \in\{0,1\} \forall i=1, \ldots, 9 ; f_{1}+\cdots+f_{9}=1\right\} .
\end{aligned}
$$

Иными словами, $\mathscr{F}_{1,1,1}$ - это семейство всевозможных вершин гиперкуба $[0,1]^{9}$, у которых в точности три координаты отличны от нуля, а $\mathscr{F}_{1}$ - это семейство всевозможных вершин того же гиперкуба, у которых есть только одна ненулевая компонента. Положим $\mathscr{F}=\mathscr{F}_{1,1,1} \cup \mathscr{F}_{1}$ и рассмотрим граф $\Gamma=(\mathscr{V}, \mathscr{E}):$ множество вершин этого графа совпадает с семейством векторов $\mathscr{F}$, а ребрами соединены те и только те вершины, расстояние между которыми равно 2. Таким образом,

$$
\mathscr{V}=\mathscr{F}, \quad \mathscr{E}=\left\{\left(F_{1}, F_{2}\right) \in \mathscr{F} \times \mathscr{F}: d\left(F_{1}, F_{2}\right)=2\right\} .
$$

Теперь, как нетрудно видеть из определения хроматического числа пространства, для доказательства оценки $\chi\left(\mathbb{Q}^{9}\right) \geqslant 12$ достаточно показать, что $\chi(\Gamma) \geqslant 12$ (здесь $\chi(\Gamma)$ - хроматическое число графа $\Gamma)$. В самом деле, рассмотрим граф $Г$ как подмножество в $\mathbb{R}^{9}$. Тогда, очевидно, что для $\Gamma^{\prime}=\frac{1}{2} \Gamma$ выполнены следующие утверждения: $\chi\left(\Gamma^{\prime}\right)=\chi(\Gamma)$ и $\Gamma^{\prime} \subset G_{1}$, где $G_{1}-$ граф̆, с помошью которого давалось определение 
хроматического числа рационального пространства. Стало быть, $\chi\left(\mathbb{Q}^{9}\right)=\chi\left(G_{1}\right) \geqslant$ $\chi\left(\Gamma^{\prime}\right)=\chi(\Gamma)$. Докажем, что $\chi(\Gamma) \geqslant 12$.

Предположим противное: $\chi(\Gamma) \leqslant 11$. Тогда семейству векторов $\mathscr{F}$ поставим во взаимно однозначное соответствие совокупность множеств $\mathscr{M}=\mathscr{M}_{1,1,1} \cup \mathscr{M}_{1} \subset 2^{\Re_{9}}$, где $\Re_{n}=\{1, \ldots, n\}$ - произвольное множество из $n$ элементов, $\mathscr{M}_{1,1,1}-$ совокупность всевозможных трехэлементных подмножеств множества $\Re_{9}$, а $\mathscr{M}_{1}$ - совокупность всевозможных одноэлементных подмножеств того же множества. Ясно тем самьм, что card $\mathscr{M}_{1,1,1}=C_{9}^{3}=84$, a card $\mathscr{M}_{1}=9$. Понятно, кроме того, что если векторам $F_{1}, F_{2} \in \mathscr{F}$ отвечают множества $M_{1}, M_{2} \in \mathscr{M}_{1,1,1}$ (т.е. $F_{1}, F_{2} \in \mathscr{F}_{1,1,1}$ ), то равенство $d\left(F_{1}, F_{2}\right)=2$ выполнено тогда и только тогда, когда $\operatorname{card}\left(M_{1} \cap M_{2}\right)=1$. В то же время, если векторам $F_{1}, F_{2} \in \mathscr{F}$ отвечают множества $M_{1} \in \mathscr{M}_{1,1,1}, M_{2} \in \mathscr{M}_{1}$ (т.е. $\left.F_{1} \in \mathscr{F}_{1,1,1}, F_{2} \in \mathscr{F}_{1}\right)$, то равенство $d\left(F_{1}, F_{2}\right)=2$ эквивалентно условию $M_{1} \cap M_{2}=\varnothing$. Значит, наше предположение о том, что $\chi(\Gamma) \leqslant 11$, равносильно, в свою очередь, предположению, что совокупность множеств $\mathscr{M}$ может быть представлена в виде

$$
\mathscr{M}=\mathscr{M}^{1} \sqcup \cdots \sqcup \mathscr{M}^{11},
$$

причем, во-первых, для любого $i, 1 \leqslant i \leqslant 11$, и любых двух множеств $M_{1}, M_{2} \in \mathscr{M}^{i} \cap$ $\mathscr{M}_{1,1,1}$ выполнено $\operatorname{card}\left(M_{1} \cap M_{2}\right) \neq 1$, а во-вторых, для любого $i, 1 \leqslant i \leqslant 11$, и любых двух множеств $M_{1} \in \mathscr{M}^{i} \cap \mathscr{M}_{1,1,1}, M_{2} \in \mathscr{M}^{i} \cap \mathscr{M}_{1}$ вьполнено $M_{2} \subset M_{1}$. Покажем, что такое разбиение невозможно. Для этого рассмотрим два случая.

Случай 1. Пусть для некоторого $j \operatorname{card}\left(\mathscr{M}^{j} \cap \mathscr{M}_{1}\right) \geqslant 3$. Тогда $\operatorname{card}\left(\mathscr{M}^{j} \cap \mathscr{M}_{1,1,1}\right)$ $\leqslant 1$, поскольку мы знаем, что для любых двух множеств $M_{1} \in \mathscr{M}^{j} \cap \mathscr{M}_{1,1,1}$, $M_{2} \in \mathscr{M}^{j} \cap \mathscr{M}_{1}$ выполнено $M_{2} \subset M_{1}$ и что card $M_{1}=3$. С другой стороны, имеет место неравенство $\operatorname{card}\left(\mathscr{M}^{i} \cap \mathscr{M}_{1,1,1}\right) \leqslant 8 \forall i=1, \ldots, 11$. (Это неравенство есть частньй случай леммы П. Эрдеша и В. Шош, доказательство которой можно найти в работе [65].) Следовательно,

$$
\begin{aligned}
84 & =\operatorname{card} \mathscr{M}_{1,1,1}=\operatorname{card}\left(\mathscr{M} \cap \mathscr{M}_{1,1,1}\right) \\
& =\operatorname{card}\left(\mathscr{M}^{j} \cap \mathscr{M}_{1,1,1}\right)+\sum_{i \neq j} \operatorname{card}\left(\mathscr{M}^{i} \cap \mathscr{M}_{1,1,1}\right) \leqslant 1+8 \times 10=81 .
\end{aligned}
$$

Полученная оценка противоречива, и, стало быть, в случае 1 разбиение (1) невозможно.

Случай 2. Пусть, наоборот, для каждого $i=1, \ldots, 11 \operatorname{card}\left(\mathscr{M}^{i} \cap \mathscr{M}_{1}\right) \leqslant 2$. Тогда равенство card $\mathscr{M}_{1}=9$ влечет наличие по меньшей мере пяти элементов разбиения (1), в каждом из которых есть хотя бы одно множество из совокупности $\mathscr{M}_{1}$. Без ограничения общности можно считать, что, например, при $j=1, \ldots, 5 \mathscr{M}^{j} \cap \mathscr{M}_{1} \neq \varnothing$. Докажем, что тем самым $\operatorname{card}\left(\mathscr{M}^{j} \cap \mathscr{M}_{1,1,1}\right) \leqslant 7 \forall j=1, \ldots, 5$. В самом деле, зафиксируем произвольное $j$ и предположим сначала, что мощность пересечения совокупности множеств $\mathscr{M}^{j}$ и совокупности множеств $\mathscr{M}_{1}$ в точности равна двум. Положим $\left\{M_{1}, M_{2}\right\}=\mathscr{M}^{j} \cap \mathscr{M}_{1}$. Тогда из условий, которым удовлетворяют элементы разбиения (1), следует, что $\left(M_{1} \cup M_{2}\right) \subset M$ для всякого $M \in \mathscr{M}^{j} \cap \mathscr{M}_{1,1,1}$. Таким образом, в сделанных предположениях оценка $\operatorname{card}\left(\mathscr{M}^{j} \cap \mathscr{M}_{1,1,1}\right) \leqslant 7$ очевидна. Предположим теперь, что $\operatorname{card}\left(\mathscr{M}^{j} \cap \mathscr{M}_{1}\right)=1$, т.е. $\mathscr{M}^{j} \cap \mathscr{M}_{1}=\left\{M_{1}\right\}$. Тогда опять-таки $M_{1} \subset M$ для всякого $M \in \mathscr{M}^{j} \cap \mathscr{M}_{1,1,1}$. С учетом условий, которым удовлетворяют элементы разбиения (1), это означает, что $\operatorname{card}\left(\mathscr{M}^{j} \cap \mathscr{M}_{1,1,1}\right) \leqslant \operatorname{card} \widetilde{\mathscr{M}}$, где $\widetilde{\mathscr{M}} \subset 2^{\Re_{8}}$ - произвольная совокупность двухэлементных подмножеств восьмиэлементного множества 
такая, что $\widetilde{M}_{1} \cap \widetilde{M}_{2} \neq \varnothing \forall \widetilde{M}_{1}, \widetilde{M}_{2} \in \widetilde{\mathscr{M}}$. Но мощность всякой такой совокупности, в свою очередь, не превосходит семи (это может быть доказано как непосредственно, так и со ссылкой на теорему Эрдеша-Ко-Радо (см. [80])). Стало быть, оценка $\operatorname{card}\left(\mathscr{M}^{j} \cap \mathscr{M}_{1,1,1}\right) \leqslant 7$ доказана полностью.

Как и в случае 1 , мы можем теперь написать неравенство:

$$
\begin{aligned}
84 & =\operatorname{card} \mathscr{M}_{1,1,1}=\operatorname{card}\left(\mathscr{M} \cap \mathscr{M}_{1,1,1}\right) \\
& =\sum_{j=1}^{5} \operatorname{card}\left(\mathscr{M}^{j} \cap \mathscr{M}_{1,1,1}\right)+\sum_{i>5} \operatorname{card}\left(\mathscr{M}^{i} \cap \mathscr{M}_{1,1,1}\right) \leqslant 7 \times 5+8 \times 6=83 .
\end{aligned}
$$

Это неравенство также противоречиво, а значит, и в случае 2 разбиение (1) невозможHо.

Ясно, что двумя рассмотренными случаями исчерпывается множество всех допустимых ситуаций, так что совокупность множеств $\mathscr{M}$ не может быть разбита на 11 частей, удовлетворяющих известным условиям, и, следовательно, наше предположение о том, что $\chi(\Gamma) \leqslant 11$, неверно. Значит, $\chi\left(\mathbb{Q}^{9}\right) \geqslant \chi(\Gamma) \geqslant 12$. Утверждение доказано полностью.

2. Перейдем теперь к изучению вешественного случая и докажем сперва, что, в самом деле, $\chi\left(\mathbb{R}^{7}\right) \geqslant 15$. С этой целью определим в пространстве $\mathbb{R}^{8}$ три семейства векторов - $\mathscr{F}_{1}, \mathscr{F}_{2}$ и $\mathscr{F}_{1,-1}$ - следуюшим образом:

$$
\begin{aligned}
\mathscr{F}_{1} & =\left\{F=\left(f_{1}, \ldots, f_{8}\right): f_{i} \in\{0,1\} \forall i=1, \ldots, 8 ; f_{1}+\cdots+f_{8}=4\right\}, \\
\mathscr{F}_{2} & =\left\{F=\left(f_{1}, \ldots, f_{8}\right): f_{i} \in\{0,2\} \forall i=1, \ldots, 8 ; f_{1}+\cdots+f_{8}=4\right\}, \\
\mathscr{F}_{1,-1} & =\left\{F=\left(f_{1}, \ldots, f_{8}\right): f_{i} \in\{1,-1\} \forall i=1, \ldots, 8 ; f_{1}+\cdots+f_{8}=4\right\} .
\end{aligned}
$$

Иными словами, $\mathscr{F}_{1}$ - это семейство всевозможных вершин гиперкуба $[0,1]^{8}$, у которых ровно четыре координаты отличны от нуля, $\mathscr{F}_{2}$ - это семейство всевозможных вершин гиперкуба $[0,2]^{8}$, у которых имеется в точности две ненулевые координаты, а $\mathscr{F}_{1,-1}$ - это семейство всевозможных вершин гиперкуба $[-1,1]^{8}$ с шестью положительными и двумя отрицательньми компонентами. Положим, как и в первом пункте настояшего раздела, $\mathscr{F}=\mathscr{F}_{1} \cup \mathscr{F}_{2} \cup \mathscr{F}_{1,-1}$ и рассмотрим граф̆ $\Gamma=(\mathscr{V}, \mathscr{E})$ следуюшего вида:

$$
\mathscr{V}=\mathscr{F}, \quad \mathscr{E}=\left\{\left(F_{1}, F_{2}\right) \in \mathscr{F} \times \mathscr{F}: d\left(F_{1}, F_{2}\right)=\sqrt{8}\right\},
$$

так что граф Г аналогичен по построению одноименному графиу из первого пункта раздела. Стало быть, и теперь $\chi\left(\mathbb{R}^{8}\right) \geqslant \chi(\Gamma)$. Более того, размерность аффинного подпространства, в котором лежит семейство векторов $\mathscr{F} \subset \mathbb{R}^{8}$, равна семи, поскольку для всякого вектора $F=\left(f_{1}, \ldots, f_{8}\right) \in \mathscr{F}$ имеет место тождество $f_{1}+\cdots+f_{8}=4$. Понятно тогда, что даже $\chi\left(\mathbb{R}^{7}\right) \geqslant \chi(\Gamma)$. Докажем, что $\chi(\Gamma) \geqslant 15$.

Предположим противное: $\chi(\Gamma) \leqslant 14$. Сопоставим тогда (взаимно однозначным образом) семейству векторов $\mathscr{F}$ совокупность множеств $\mathscr{M}=\mathscr{M}_{1} \cup \mathscr{M}_{2} \cup \mathscr{M}_{1,-1} \subset$ $2^{\Re}$, где $\mathscr{M}_{1}$ - совокупность всевозможных четырехэлементных подмножеств множества $\Re_{8}, \mathscr{M}_{2}$ - совокупность всевозможных двухэлементных подмножеств множества $\Re_{8}$, а $\mathscr{M}_{1,-1}$ - совокупность всевозможных шестиэлементных подмножеств того же множества. Ясно, таким образом, что $\operatorname{card} \mathscr{M}_{1}=C_{8}^{4}=70, \operatorname{card} \mathscr{M}_{2}=C_{8}^{2}=28$, а card $\mathscr{M}_{1,-1}=C_{8}^{6}=C_{8}^{2}=28$. Понятно также, что, как и в п. 1 настоящего раздела, 
наше предположение о том, что $\chi(\Gamma) \leqslant 14$, равносильно, в свою очередь, допушению, что совокупность множеств $\mathscr{M}$ может быть представлена в виде

$$
\mathscr{M}=\mathscr{M}^{1} \sqcup \cdots \sqcup \mathscr{M}^{14}
$$

причем для каждого $i=1, \ldots, 14$ имеют место следующие свойства.

Свойство 1. Если $M_{1}, M_{2} \in \mathscr{M}^{i} \cap \mathscr{M}_{1}$, то $M_{1} \cap M_{2} \neq \varnothing$.

Свойство 2. Если $M_{1}, M_{2} \in \mathscr{M}^{i} \cap \mathscr{M}_{2}$, то $M_{1} \cap M_{2}=\varnothing$.

Свойство 3. Если $M_{1}, M_{2} \in \mathscr{M}^{i} \cap \mathscr{M}_{1,-1}$, то саrd $\left(M_{1} \cap M_{2}\right)=4$, т.е., иначе говоря, $\widetilde{M}_{1} \cap \widetilde{M}_{2}=\varnothing$, где $\widetilde{M}_{i}=\Re_{8} \backslash M_{i}$.

Свойство 4. Если $M_{1} \in \mathscr{M}^{i} \cap \mathscr{M}_{1}$, а $M_{2} \in \mathscr{M}^{i} \cap \mathscr{M}_{2}$, то $\operatorname{card}\left(M_{1} \cap M_{2}\right) \neq 1$.

Свойство 5. Если $M_{1} \in \mathscr{M}^{i} \cap \mathscr{M}_{1}$, а $M_{2} \in \mathscr{M}^{i} \cap \mathscr{M}_{1,-1}$, то $\operatorname{card}\left(M_{1} \cap M_{2}\right) \neq 3$.

Свойство 6. Если $M_{1} \in \mathscr{M}^{i} \cap \mathscr{M}_{2}$, а $M_{2} \in \mathscr{M}^{i} \cap \mathscr{M}_{1,-1}$, то $M_{1} \not \subset M_{2}$.

Покажем, что, на самом деле, разбиение (2) не может удовлетворять всем свойствам 1-6. Сначала заметим, что в работе [65] была фактически получена оценка:

$$
\operatorname{card}\left(\mathscr{M}^{i} \cap\left(\mathscr{M}_{2} \cup \mathscr{M}_{1,-1}\right)\right) \leqslant 4, \quad i=1, \ldots, 14 .
$$

Но тогда ясно, что в нашей ситуации

$$
\operatorname{card}\left(\mathscr{M}^{i} \cap\left(\mathscr{M}_{2} \cup \mathscr{M}_{1,-1}\right)\right)=4, \quad i=1, \ldots, 14,
$$

поскольку в противном случае имело бы место следующее противоречивое неравенство:

$$
56=\operatorname{card}\left(\mathscr{M}_{2} \cup \mathscr{M}_{1,-1}\right)=\operatorname{card}\left(\mathscr{M} \cap\left(\mathscr{M}_{2} \cup \mathscr{M}_{1,-1}\right)\right) \leqslant 3+13 \times 4=55 .
$$

Следовательно,

$$
\mathscr{M}^{i} \cap\left(\mathscr{M}_{2} \cup \mathscr{M}_{1,-1}\right)=\left\{M_{1}^{i}, M_{2}^{i}, M_{3}^{i}, M_{4}^{i}\right\}, \quad i=1, \ldots, 14,
$$

и для каждого $i$ необходимо рассмотреть ровно пять случаев, а именно: 1) все четыре множества $M_{j}^{i}$ принадлежат совокупности $\left.\mathscr{M}_{2} ; 2\right)$ три множества $M_{j_{1}}^{i}, M_{j_{2}}^{i}, M_{j_{3}}^{i}$ с некоторыми $j_{1}, j_{2}, j_{3}$ принадлежат совокупности $\mathscr{M}_{2}$, а четвертое множество лежит в совокупности $\left.\mathscr{M}_{1,-1} ; 3\right)$ в каждой из совокупностей $\mathscr{M}_{2}$ и $\mathscr{M}_{1,-1}$ есть ровно по два множества вида $\left.M_{j}^{i} ; 4\right)$ три множества $M_{j_{1}}^{i}, M_{j_{2}}^{i}, M_{j_{3}}^{i}$ с некоторыми $j_{1}, j_{2}, j_{3}$ принадлежат совокупности $\mathscr{M}_{1,-1}$, а четвертое множество лежит в совокупности $\left.\mathscr{M}_{2} ; 5\right)$ все четыре множества $M_{j}^{i}$ принадлежат совокупности $\mathscr{M}_{1,-1}$. С лучаи 2 ) и 4 ) отпадают сразу, поскольку они несовместимы со свойствами 6 и 2 и свойствами 6 и 3 соответственно. В самом деле, рассмотрим, скажем, случай 2) (случай 4) рассматривается аналогично). Будем считать без ограничения общности, что $M_{1}^{i}, M_{2}^{i}, M_{3}^{i} \in \mathscr{M}_{2}$, а $M_{4}^{i} \in \mathscr{M}_{1,-1}$. Тогда в силу свойства 2 опять-таки без ограничения обшности $M_{1}^{i}=\{1,2\}, M_{2}^{i}=$ $\{3,4\}, M_{3}^{i}=\{5,6\}$. Но с учетом свойства $66=\operatorname{card} M_{4}^{i}=\operatorname{card}\left(\{1,2\} \cap M_{4}^{i}\right)+\cdots+$ $\operatorname{card}\left(\{7,8\} \cap M_{4}^{i}\right) \leqslant 3 \times 1+2=5$, что невозможно.

Итак, остаются только первьй, третий и пятьй случаи. Докажем, что при любом значении $i, 1 \leqslant i \leqslant 14$, в каждом из этих случаев вьполняется оценка $\operatorname{card}\left(\mathscr{M}^{i} \cap \mathscr{M}_{1}\right) \leqslant 3$. Зафиксируем некоторое $i$, например, $i=1$.

Случай 1). Здесь мы предполагаем, что $M_{1}^{1}, \ldots, M_{4}^{1} \in \mathscr{M}_{2}$. Тогда из свойства 2 следует, что без ограничения общности $M_{1}^{1}=\{1,2\}, M_{2}^{1}=\{3,4\}, M_{3}^{1}=\{5,6\}$, 
$M_{4}^{1}=\{7,8\}$. Далее, из свойства 4 видно, что любое множество $M \in \mathscr{M}^{1} \cap \mathscr{M}_{1}-$ это либо $\{1,2,3,4\}$, либо $\{1,2,5,6\}$, либо $\{1,2,7,8\}$ и т. д. Наконец, из свойства 1 и теоремы Эрдеша-Ко-Радо (см. [80]) немедленно получается необходимая нам оценка: $\operatorname{card}\left(\mathscr{M}^{1} \cap \mathscr{M}_{1}\right) \leqslant 3$.

Случай 3). Здесь мы предполагаем без ограничения обшности, что $M_{1}^{1}, M_{2}^{1} \in \mathscr{M}_{2}$, а $M_{3}^{1}, M_{4}^{1} \in \mathscr{M}_{1,-1}$. Тогда из свойств 2,3 и 6 следует, что единственная допустимая ситуация эквивалентна следующей: $M_{1}^{1}=\{1,2\}, M_{2}^{1}=\{3,4\}, \widetilde{M}_{3}^{1}=\{1,3\}, \widetilde{M}_{4}^{1}=$ $\{2,4\}$. (Здесь $\widetilde{M}_{j}^{1}=\Re_{8} \backslash M_{j}^{1}, j=3,4$.) В свою очередь, из свойств 1,4 и 5 получается, что множество $M \in \mathscr{M}^{1} \cap \mathscr{M}_{1}$ - это либо $\{1,2,3,4\}$, либо $\{5,6,7,8\}$, так что и в этом случае искомая оценка имеет место.

Наконец, случай 5) полностью аналогичен случаю 1) с той лишь разницей, что теперь следует ссылаться не на свойства 2 и 4 , а на свойства 3 и 5 . Стало быть, оценка $\operatorname{card}\left(\mathscr{M}^{i} \cap \mathscr{M}_{1}\right) \leqslant 3$ доказана полностью и для любого $i$. Коль скоро эта оценка верна, имеет место неравенство

$$
70=\operatorname{card} \mathscr{M}_{1}=\operatorname{card}\left(\mathscr{M} \cap \mathscr{M}_{1}\right)=\sum_{i=1}^{14} \operatorname{card}\left(\mathscr{M}^{i} \cap \mathscr{M}_{1}\right) \leqslant 3 \times 14=42 .
$$

Однако это неравенство противоречиво, и, значит, наше предположение о возможности построения разбиения (2) со свойствами 1-6 неверно. Следовательно, неверно и предположение о том, что $\chi(\Gamma) \leqslant 14$. Таким образом, $\chi\left(\mathbb{R}^{7}\right) \geqslant \chi(\Gamma) \geqslant 15$ и наше утверждение доказано полностью.

ЗАмечАниЕ. Прежде чем переходить к изложению доказательства оценки $\chi\left(\mathbb{R}^{11}\right) \geqslant 20$, следует отметить, что конструкция, представленная в п. 2 настоящего раздела, является по сушеству вещественной, т.е. конструкция эта не позволяет получать аналогичные оценки для рационального случая. Это связано с тем, что, во-первых, для рационального случая не проходит “трюк” с понижением размерности, а во-вторых, в упомянутой конструкции роль критического расстояния играло иррациональное число. Однако, конечно же, полученные результаты могут быть использованы в оценках $\chi\left(\mathbb{Q}^{8}, \sqrt{2}\right) \geqslant 15$ и $\chi\left(\mathbb{Q}^{8}(\sqrt{2})\right) \geqslant 15$ (здесь $\mathbb{Q}^{8}(\sqrt{2})=\mathbb{Q}(\sqrt{2}) \times \cdots \times \mathbb{Q}(\sqrt{2})$, а $\mathbb{Q}(\sqrt{2})$ - расширение поля $\left.\mathbb{Q}\right)$. Подобные задачи были, в частности, рассмотрены в работе Бенды и Перлеса (см. [51] и [64]).

3. Докажем, наконец, что $\chi\left(\mathbb{R}^{11}\right) \geqslant 20$. Для этого поступим стандартным образом: определим в пространстве $\mathbb{R}^{11}$ четыре семейства векторов - $\mathscr{F}_{1}, \mathscr{F}_{2}, \mathscr{F}_{3}$ и $\mathscr{F}_{1+\sqrt{2}}$ :

$$
\begin{aligned}
& \mathscr{F}_{1}=\left\{F=\left(f_{1}, \ldots, f_{11}\right): f_{i} \in\{0,1\} \forall i=1, \ldots, 11 ; f_{1}+\cdots+f_{11}=1\right\}, \\
& \mathscr{F}_{2}=\left\{F=\left(f_{1}, \ldots, f_{11}\right): f_{i} \in\left\{0, \frac{1+\sqrt{3}}{2}\right\} \forall i=1, \ldots, 11 ;\right. \\
&\left.f_{1}+\cdots+f_{11}=1+\sqrt{3}\right\}, \\
& \mathscr{F}_{3}=\left\{F=\left(f_{1}, \ldots, f_{11}\right): f_{i} \in\{0,1\} \forall i=1, \ldots, 11 ; f_{1}+\cdots+f_{11}=3\right\}, \\
& \mathscr{F}_{1+\sqrt{2}}=\left\{F=\left(f_{1}, \ldots, f_{11}\right): f_{i} \in\{0,1+\sqrt{2}\} \forall i=1, \ldots, 11 ;\right. \\
&\left.f_{1}+\cdots+f_{11}=1+\sqrt{2}\right\} .
\end{aligned}
$$

Определим, далее, семейство векторов $\mathscr{F}=\mathscr{F}_{1} \cup \mathscr{F}_{2} \cup \mathscr{F}_{3} \cup \mathscr{F}_{1+\sqrt{2}}$ и рассмотрим граф $\Gamma=(\mathscr{V}, \mathscr{E})$ стандартного вида:

$$
\mathscr{V}=\mathscr{F}, \quad \mathscr{E}=\left\{\left(F_{1}, F_{2}\right) \in \mathscr{F} \times \mathscr{F}: d\left(F_{1}, F_{2}\right)=2\right\} .
$$


Таким образом, $\chi\left(\mathbb{R}^{11}\right) \geqslant \chi(\Gamma)$. Докажем, что $\chi(\Gamma) \geqslant 20$.

Как и прежде, предположим противное: $\chi(\Gamma) \leqslant 19,-$ и от семейства векторов $\mathscr{F}$ перейдем к совокупности множеств

$$
\mathscr{M}=\mathscr{M}_{1} \cup \mathscr{M}_{2} \cup \mathscr{M}_{3} \cup \mathscr{M}_{1+\sqrt{2}} \subset 2^{\Re_{11}},
$$

так что $\mathscr{M}_{1}-$ совокупность всевозможных одноэлементных подмножеств, $\mathscr{M}_{2}-$ совокупность всевозможных двухэлементных подмножеств, $\mathscr{M}_{3}-$ совокупность всевозможных трехэлементных подмножеств, а $\mathscr{M}_{1+\sqrt{2}}-$ совокупность всевозможных десятиэлементных подмножеств множества $\Re_{11}$. Тем самым card $\mathscr{M}_{1}=11$, $\operatorname{card} \mathscr{M}_{2}=$ $C_{11}^{2}=55, \operatorname{card} \mathscr{M}_{3}=C_{11}^{3}=165$, a $\operatorname{card} \mathscr{M}_{1+\sqrt{2}}=11$. В таких обозначениях наше предположение о том, что $\chi(\Gamma) \leqslant 19$, равносильно, в свою очередь, допушению, что совокупность множеств $\mathscr{M}$ может быть разложена на девятнадцать подсовокупностей:

$$
\mathscr{M}=\mathscr{M}^{1} \sqcup \cdots \sqcup \mathscr{M}^{19},
$$

причем для каждого $i=1, \ldots, 19$ имеют место следуюшие свойства.

Свойство 1. Если $M_{1}, M_{2} \in \mathscr{M}^{i} \cap \mathscr{M}_{3}$, то $\operatorname{card}\left(M_{1} \cap M_{2}\right) \neq 1$.

Свойство 2. Если $M_{1} \in \mathscr{M}^{i} \cap \mathscr{M}_{3}$, а $M_{2} \in \mathscr{M}^{i} \cap \mathscr{M}_{1}$, то $M_{2} \subset M_{1}$.

Свойство 3. Если $M_{1} \in \mathscr{M}^{i} \cap \mathscr{M}_{3}$, а $M_{2} \in \mathscr{M}^{i} \cap \mathscr{M}_{1+\sqrt{2}}$, то $M_{1} \cap \widetilde{M}_{2}=\varnothing$. (Здесь $\widetilde{M}_{2}=\Re_{11} \backslash M_{2}$.)

Свойство 4. Если $M_{1} \in \mathscr{M}^{i} \cap \mathscr{M}_{3}$, а $M_{2} \in \mathscr{M}^{i} \cap \mathscr{M}_{2}$, то $\operatorname{card}\left(M_{1} \cap M_{2}\right) \neq 1$.

Покажем, что разбиение (3) со свойствами 1-4 невозможно. Для этого нам потребуются следуюшие простые леммы и следствия из них.

Лемма 1. Без ограничения общ,ности можно считать, ито $\operatorname{card}\left(\mathscr{M}^{i} \cap \mathscr{M}_{1+\sqrt{2}}\right)$ $\geqslant 2$ при $i=1,2,3$ u $\operatorname{card}\left(\mathscr{M}^{4} \cap \mathscr{M}_{1+\sqrt{2}}\right) \geqslant 1$.

Лemma 2. Eсли $\operatorname{card}\left(\mathscr{M}^{i} \cap \mathscr{M}_{2}\right) \geqslant 4, m o \operatorname{card}\left(\mathscr{M}^{i} \cap \mathscr{M}_{3}\right) \leqslant 7$.

Лемма 3. $E с л л u \operatorname{card}\left(\mathscr{M}^{i} \cap \mathscr{M}_{2}\right) \geqslant 5, m o \operatorname{card}\left(\mathscr{M}^{i} \cap \mathscr{M}_{3}\right) \leqslant 6$.

Лемма 4. Eсли $\operatorname{card}\left(\mathscr{M}^{i} \cap \mathscr{M}_{2}\right) \geqslant 3, a \operatorname{card}\left(\mathscr{M}^{i} \cap \mathscr{M}_{1}\right) \geqslant 1, m o \operatorname{card}\left(\mathscr{M}^{i} \cap \mathscr{M}_{3}\right) \leqslant 6$.

Лемма 5. Ecли $\operatorname{card}\left(\mathscr{M}^{i} \cap \mathscr{M}_{2}\right) \geqslant 2, a \operatorname{card}\left(\mathscr{M}^{i} \cap \mathscr{M}_{1}\right) \geqslant 1, m o \operatorname{card}\left(\mathscr{M}^{i} \cap \mathscr{M}_{3}\right) \leqslant 7$.

Лемма 6. Если $\operatorname{card}\left(\mathscr{M}^{i} \cap \mathscr{M}_{2}\right) \geqslant 4, i=1, \ldots, 4$ (т.е. $i$ соответствует лемме 1$),$ mo $\operatorname{card}\left(\mathscr{M}^{i} \cap \mathscr{M}_{3}\right) \leqslant 6$.

Лемма 7. Если $\operatorname{card}\left(\mathscr{M}^{i} \cap \mathscr{M}_{2}\right) \geqslant 7, i=1, \ldots, 4, m o \operatorname{card}\left(\mathscr{M}^{i} \cap \mathscr{M}_{3}\right) \leqslant 5$.

Лемма 8. Eсли $\operatorname{card}\left(\mathscr{M}^{i} \cap \mathscr{M}_{1}\right) \geqslant 3, m o \operatorname{card}\left(\mathscr{M}^{i} \cap \mathscr{M}_{3}\right) \leqslant 1$.

СлеДСтвИЕ 1. Пусть $l \geqslant 1, \quad a \quad\left\{i_{1}, \ldots, i_{l}\right\} \cap\{1, \ldots, 4\}=\varnothing . \quad$ Тогда если $\operatorname{card}\left(\mathscr{M}^{i_{\nu}} \cap \mathscr{M}_{1}\right) \geqslant 1 \quad \forall \nu=1, \ldots, l, m o$

$$
\operatorname{card}\left(\left(\mathscr{M}^{i_{1}} \cup \cdots \cup \mathscr{M}^{i_{l}}\right) \cap \mathscr{M}_{2}\right) \leqslant l+1 .
$$

СлЕДСТВИЕ 2. Имеет место неравенство

$$
\operatorname{card}\left(\left(\mathscr{M}^{1} \cup \cdots \cup \mathscr{M}^{4}\right) \cap \mathscr{M}_{2}\right) \leqslant 15 .
$$


СлЕДСтВИЕ 3. В разбиении (3) найдется по крайней мере 6 частей, имеющих непустое пересечение с совокупностью множеств $\mathscr{M}_{1}$.

Доказательства этих лемм и следствий могут легко быть получены путем простого перебора и многократного применения свойств 1-4 и леммы Эрдеша и Шош (см. [65]), так что в настоящей работе мы эти доказательства не приводим. Заметим только, что следствие 1 вытекает из лемм 4 и 5 , следствие 2 - из лемм 6 и 7 , а следствие $3-$ из леммы 8. Рассмотрим теперь пять случаев и в каждом из них получим некоторое противоречивое неравенство.

Случай 1). В этом случае мы предполагаем, что для любого $i=1, \ldots, 4$ вьполнено $\mathscr{M}^{i} \cap \mathscr{M}_{1} \neq \varnothing$. Тогда нетрудно видеть, что из свойств $1-3$, леммы 1 , леммы Эрдеша и Шош и леммы Эрдеша-Ко-Радо следует оценка

$$
\operatorname{card}\left(\left(\mathscr{M}^{1} \cup \cdots \cup \mathscr{M}^{4}\right) \cap \mathscr{M}_{3}\right) \leqslant 29 .
$$

Но эта оценка ведет к противоречию, так как из нее и леммы Эрдеша и Шош вытекает неравенство

$$
165=\operatorname{card} \mathscr{M}_{3}=\operatorname{card}\left(\mathscr{M} \cap \mathscr{M}_{3}\right) \leqslant 29+15 \times 9=164,
$$

что невозможно. Значит, в этом случае разбиение (3) со свойствами 1-4 построено быть не может.

Случай 2). Здесь мы предполагаем, что лишш три совокупности вида $\mathscr{M}^{1}, \ldots, \mathscr{M}^{4}$ имеют непустое пересечение с совокупностью $\mathscr{M}_{1}$. Тогда выполнена оценка, аналогичная оценке (4):

$$
\operatorname{card}\left(\left(\mathscr{M}^{1} \cup \cdots \cup \mathscr{M}^{4}\right) \cap \mathscr{M}_{3}\right) \leqslant 30 .
$$

Кроме того, с учетом следствия 3 мы можем без ограничения обшности считать, что совокупности $\mathscr{M}^{5}, \mathscr{M}^{6}$ и $\mathscr{M}^{7}$ также имеют непустое пересечение с $\mathscr{M}_{1}$. Допустим сперва, что какая-нибудь из последних трех совокупностей содержит по меньшей мере два множества из $\mathscr{M}_{2}$. Тогда применима лемма 5 , которая вкупе с оценкой $(5)$ и леммой Эрдеша и Шош дает противоречивое неравенство

$$
165=\operatorname{card} \mathscr{M}_{3}=\operatorname{card}\left(\mathscr{M} \cap \mathscr{M}_{3}\right) \leqslant 30+7+14 \times 9=163 .
$$

Стало быть, сделанное нами допущение неверно и $\operatorname{card}\left(\mathscr{M}^{i} \cap \mathscr{M}_{2}\right) \leqslant 1, i=5,6,7$. Из этого наблюдения и следствия 2 следует, что $\operatorname{card}\left(\bigcup_{i=1}^{7} \mathscr{M}^{i} \cap \mathscr{M}_{2}\right) \leqslant 18$. Поскольку $\operatorname{card} \mathscr{M}_{2}=55$, это означает, что в двенадщати элементах разбиения (3) имеется по крайней мере 37 множеств из совокупности $\mathscr{M}_{2}$. По принципу Дирихле найдется один из этих элементов, в котором не меньше четырех множеств из $\mathscr{M}_{2}$. Остается применить лемму 2 и неравенство (5):

$$
165=\operatorname{card} \mathscr{M}_{3}=\operatorname{card}\left(\mathscr{M} \cap \mathscr{M}_{3}\right) \leqslant 30+7+14 \times 9=163 .
$$

Полученная оценка вновь противоречива, и, значит, в случае 2) разбиение (3) также невозможно.

Случай 3). Теперь мы считаем, что только две из совокупностей $\mathscr{M}^{1}, \ldots, \mathscr{M}^{4}$ имеют непустое пересечение с $\mathscr{M}_{1}$. Опять-таки это влечет неравенство

$$
\operatorname{card}\left(\left(\mathscr{M}^{1} \cup \cdots \cup \mathscr{M}^{4}\right) \cap \mathscr{M}_{3}\right) \leqslant 31 .
$$


Снова замечаем, что следствие 3 позволяет гарантировать наличие по меньшей мере четырех элементов разбиения $(3),-$ скажем, $\mathscr{M}^{5}, \ldots, \mathscr{M}^{8},-$ имеюших непустое пересечение с $\mathscr{M}_{1}$. В свою очередь, следствия 1 и 2 обеспечивают оценку

$$
\operatorname{card}\left(\bigcup_{i=1}^{8} \mathscr{M}^{i} \cap \mathscr{M}_{2}\right) \leqslant 20 .
$$

Стало быть, в разбиении (3) есть одиннадцать элементов, содержащих в объединении не менее 35 множеств из $\mathscr{M}_{2}$. По принципу Дирихле либо найдется один из этих элементов, в котором больше четырех множеств из $\mathscr{M}_{2}$, либо найдется два таких элемента, причем в каждом из них будет не меньше четырех множеств из $\mathscr{M}_{2}$. Учитьвая неравенство (6) и, смотря по ситуации, либо применяя лемму 3 , либо дважды ссылаясь на лемму 2 , получаем:

$$
165=\operatorname{card} \mathscr{M}_{3}=\operatorname{card}\left(\mathscr{M} \cap \mathscr{M}_{3}\right) \leqslant 31+2 \times 7+13 \times 9=162,
$$

либо

$$
165=\operatorname{card} \mathscr{M}_{3}=\operatorname{card}\left(\mathscr{M} \cap \mathscr{M}_{3}\right) \leqslant 31+6+14 \times 9=163,
$$

что одинаково противоречиво. Тем самым и в случае 3 ) разбиение (3) невозможно.

Нетрудно понять теперь, что представляют из себя оставшиеся два случая. Более того, рассмотрение их полностью аналогично рассмотрению случая 3 ), и посему в настояшей работе мы его не проводим. В то же время ясно, что пятью такими случаями исчерпываются все допустимые ситуации. Следовательно, наше предположение о возможности построения разбиения (3), удовлетворяющего свойствам 1-4, неверно и $\chi\left(\mathbb{R}^{11}\right) \geqslant 20$.

ЗАмЕчаниЕ 1. Нетрудно видеть, что, как и в п. 2 настоящего раздела, конструкция, предложенная для доказательства оценки $\chi\left(\mathbb{R}^{11}\right) \geqslant 20$, является существенно нерациональной, поскольку на сей раз в ней присутствуют векторы с иррациональными координатами. Однако, конечно же, эта конструкция может быть применена к доказательству оценки $\chi\left(\mathbb{Q}^{11}(\sqrt{2}, \sqrt{3})\right) \geqslant 20$, т.е. опять-таки мы имеем дело с метрическими пространствами, изученными, в частности, в работах [51] и [64].

ЗАмЕчАниЕ 2. В заключение следует дать определение так назьваемой $(M, D)$ критической конфигурации: говорят, что конечное множество векторов $\mathscr{F}$ из метрического пространства $(X, r)$ образует $(M, D)$-критическую конфигурацию с критическим расстоянием $a>0$, если саrd $\mathscr{F}=M$ и в любом множестве $\mathscr{F}^{\prime} \subset \mathscr{F}$, $\operatorname{card} \mathscr{F}^{\prime}=D+1$, найдется пара векторов $F_{1}, F_{2} \in \mathscr{F}^{\prime}$ таких, что $r\left(F_{1}, F_{2}\right)=a$. Ясно тогда, что по принщипу Дирихле $\chi(X, a) \geqslant[M / D]+1$ (ср. аналогичные утверждения в начале каждого из пунктов настоящего раздела). Определение $(M, D)$-критической конфигурации для случая, когда $(X, r)$ - это $\mathbb{R}^{d}$ с евклидовой метрикой, было дано Ларманом и Роджерсом в 1972 году в работе [65], и практически все нижние оценки для $\chi\left(\mathbb{R}^{d}\right)$ и $\chi\left(\mathbb{Q}^{d}\right)$, перечисленные в разделах 3.2 и 3.3 , были получены как раз с помошю построения некоторой $(M, D)$-критической конфигурации с достаточно большим отношением $M / D$ (см. [38], [59], [61], [63], [65], [66], [67], [70], [71], [74] и [75]). Исключение составляют лиш доказанные в этом разделе оценки. В самом деле, если мы рассмотрим семейство векторов $\mathscr{F}$ из первого пункта настоящего раздела, то для него $M=93$, а $D \geqslant 9$, так что $[M / D]+1 \leqslant 11$, в то время как мы доказали оценку 
$\chi\left(\mathbb{Q}^{9}\right) \geqslant 12$. Аналогично во втором пункте $M=126, D \geqslant 35$, и $[M / D]+1 \leqslant 4$, тогда как у нас $\chi\left(\mathbb{R}^{7}\right) \geqslant 15$. Наконец, в третьем пункте $M=242, D \geqslant 77$, и $[M / D]+1 \leqslant 4$, что опять-таки несопоставимо с оценкой $\chi\left(\mathbb{R}^{11}\right) \geqslant 20$.

4.2. Асимптотические оценки величин $f(d), \chi\left(\mathbb{R}^{d}\right), \chi\left(\mathbb{Q}^{d}\right), \chi\left(\mathbb{R}^{d}, a_{1}, \ldots, a_{k}\right)$. В этом разделе мы сформулируем обший метод получения нижних оценок для минимального числа частей меньшего диаметра и для хроматических чисел вешественных и рациональных пространств. С помощью этого метода мы получим различные оценки, приведенные в параграфах 2 и 3.

ОПРЕДЕлЕнИЕ. Многогранник $\mathscr{P} \subset \mathbb{R}^{d}$ мы будем называть целочисленным, если он может быть получен в качестве выпуклой оболочки некоторого множества целых точек $\mathbf{x}_{1}, \ldots, \mathbf{x}_{s} \in \mathbb{Z}^{d}: \mathscr{P}=\operatorname{conv}\left\{\mathbf{x}_{1}, \ldots, \mathbf{x}_{s}\right\}$. Само же множество точек $\left\{\mathbf{x}_{1}, \ldots, \mathbf{x}_{s}\right\}$ мы будем назьвать определяющей системой точек для целочисленного многогранника $\mathscr{P}$. В случае, когда определяюшая система точек содержится в множестве вершин гиперкуба $[0,1]^{d}$, соответствуюший целочисленньй многогранник $\mathscr{P}$ назьвается $(0,1)$-многогранником; если же координаты точек определяюшей системы могут принимать три значения - 0,1 и $-1,-$ то соответствующий целочисленньй многогранник называется $(0,1,-1)$-многогранником или кросс-политопом.

В соответствии с данным определением упомянутый общий метод формулируется следуюшим образом.

ОБщий метоД. Для получения нижних оценок величины $f(d)$ следует подобрать некоторьй целочисленный многогранник (или, что то же самое, его определяющую систему), требуюший (требующую) при разбиении на части меньшего диаметра достаточно большого числа таких частей. Для получения нижних оценок хроматических чисел вешественных и рациональных пространств следует также подобрать некоторую определяюшую систему точек для целочисленного многогранника и рассмотреть эту систему как $(M, D)$-критическую конфигурацию (см. замечание 2 в раздел 4.1).

Итак, суть сформулированного метода состоит в том, что для решения интересующих нас задач оказывается удобно работать с некоторыми (конечными) наборами точек целочисленной решетки $\mathbb{Z}^{d}$, интерпретируя их, в зависимости от ситуации, либо как определяюшие системы для целочисленных многогранников, либо как $(M, D)$-критические конфигурации.

Покажем теперь, как с помощью сформулированного нами метода получать различные оценки для величин $f(d), \chi\left(\mathbb{R}^{d}\right)$ и $\chi\left(\mathbb{Q}^{d}\right)$.

ЗАмечАниЕ. Строго говоря, только что сформулированный нами общий метод не позволяет работать с обобшенньми хроматическими числами, т.е. получать нижние оценки для величины $\chi\left(\mathbb{R}^{d}, a_{1}, \ldots, a_{k}\right)$ при $k \geqslant 2$. Однако ситуацию очень легко исправить, дав следуюшее (общее) определение.

ОПреДЕЛЕНИЕ. Конечное множество векторов $\mathscr{F}$ из метрического пространства $(X, r)$ назьвается $(M, D, k)$-критической конфигурацией с критическими (запрешенными) расстояниями $a_{1}>0, \ldots, a_{k}>0$, если card $\mathscr{F}=M$ и в любом множестве $\mathscr{F}^{\prime} \subset \mathscr{F}, \operatorname{card} \mathscr{F}^{\prime}=D+1$, найдется пара векторов $F_{1}, F_{2} \in \mathscr{F}^{\prime}$ таких, что $r\left(F_{1}, F_{2}\right) \in$ $\left\{a_{1}, \ldots, a_{k}\right\}$. 
Ясно тогда, что, как и при рассмотрении обычных $(M, D)$-критических конфигураций, по принципу Дирихле может быть получена оценка $\chi\left(X, a_{1}, \ldots, a_{k}\right) \geqslant M / D$, при условии, конечно, что нам удалось в пространстве $X$ построить некоторую $(M, D, k)$ критическую конфигурацию с запрешенньми расстояниями $a_{1}, \ldots, a_{k}$. Понятно, стало быть, как надо видоизменить формулировку обшего метода, дабы он (хотя бы формально) оказался применим к задаче нахождения нижних оценок для обобщенных хроматических чисел. В дальнейшем мы покажем, что такой метод, действительно, может быть весьма эффективно применен к величине $\chi\left(\mathbb{R}^{d}, a_{1}, \ldots, a_{k}\right)$.

1. Доказательство оценки Кана и Калаи $f(d) \geqslant(1.203 \ldots+o(1))^{\sqrt{d}}$ (см. [39]). В 1981 году П. Франкл и Р. Вилсон доказали, в частности, следующую теорему (см. [38]).

ТЕОРемА ФРАнКЛА-ВИЛСОНА. Пусть $q=p^{\alpha}$ - некоторая степень простого числа р. Пусть, далее, $\mathscr{M}$ - некоторая совокупность $k$-элементных подмножеств $n$-элементного множества таких, что мощность пересечения любых двух из них не сравнима с $k$ по модулю $q$. Тогда $\operatorname{card} \mathscr{M} \leqslant C_{n}^{q-1}$.

СледСТВИЕ. Пусть $q=p^{\alpha}-$ некоторая степень простого числа $p$. Положим $n=4 q$. Пусть $\mathscr{M}$ - некоторая совокупность $\frac{n}{2}$-әлементных подмножеств множества $\Re_{n}=\{1, \ldots, n\}$, причем для любых двух множеств $M_{1}, M_{2} \in \mathscr{M}$ выполнено $\operatorname{card}\left(M_{1} \cap M_{2}\right) \neq \frac{n}{4}$. Тогда $\operatorname{card} \mathscr{M} \leqslant 2 C_{n-1}^{n / 4-1}$.

ЗАмечАниЕ 1. Суть теоремы Франкла-Вилсона и следствия из нее состоит в том, что иногда в совокупности множеств достаточно всего лишь одного запрета на возможные мошности попарных пересечений множеств из этой совокупности для существенного понижения верхней оценки на максимально достижимую мощность такой совокупности: например, если в следствии опустить условие $\operatorname{card}\left(M_{1} \cap M_{2}\right) \neq \frac{n}{4}$, то в этом случае величина $\operatorname{card} \mathscr{M}$ с очевидностью сможет принимать значение $C_{n}^{n / 2}$; однако $2 C_{n-1}^{n / 4-1}=(1.7 \ldots+o(1))^{n}$, в то время как $C_{n}^{n / 2}=(2+o(1))^{n}$, т.е. единственное условие, наложенное на мошности попарных пересечений множеств в совокупности, немедленно влечет экспоненциальное понижение оценки мощности самой этой совокупности.

ЗАмечАние 2. Доказательство теоремы Франкла-Вилсона и следствия из нее основано на применении методов линейной алгебры в комбинаторике (см. [38], [75] и [81]), и в настоящей работе мы его не приводим. (Впрочем, доказательство теоремы Франкла-Вилсона может быть получено и с помошью некоторой модификации линейно-алгебраических методов, которые будут использованы в настоящей работе, см., скажем, п. 2 текушего раздела. Тем не менее мы на этих модификациях останавливаться не будем.) Заметим также, что некоторые схожие результаты и полезные ссылки могут быть найдены в работах [82]-[84].

Рассмотрим множество $\Re_{n}$ с $n=4 q$, где $q$, как и в теореме Франкла-Вилсона, степень некоторого простого числа. Определим $W$ как множество всех пар элементов из $\Re_{n}$. Если $\Re_{n}=A \sqcup B$, то положим $S(A, B)=\{w \in W: \operatorname{card}(w \cap A)=1$; $\operatorname{card}(w \cap B)=1\}$. Пусть, наконец, $\mathscr{M}=\{S(A, B): \operatorname{card} A=2 q\}$. Тогда $\mathscr{M}$ можно интерпретировать как некоторую совокупность $\frac{n^{2}}{4}$-элементных подмножеств множества $\Re_{n(n-1) / 2}$, причем card $\mathscr{M}=\frac{1}{2} C_{n}^{n / 2}$. Заметим, что пересечение множеств $S(A, B)$ и $S(C, D)$ имеет наименьшую мошность тогда и только тогда, когда $\operatorname{card}(A \cap C)=q$. 
Стало быть, по следствию из теоремы Франкла-Вилсона в любой подсовокупности $\mathscr{L} \subset \mathscr{M}$ с условием card $\mathscr{L}>2 C_{n-1}^{n / 4-1}$ найдется пара множеств с наименьшей мошностью пересечения. В то же время ясно, что совокупность множеств $\mathscr{M}$ можно естественным образом проинтерпретировать как определяющую систему точек для некоторого $(0,1)$-многогранника, причем диаметр этого многогранника будет достигаться как раз на тех парах точек из определяющей системы, которые отвечают множествам с наименьшей мощностью пересечения. Значит, по принципу Дирихле такой $(0,1)$-многогранник не может быть разбит на меньше чем

$$
\frac{\frac{1}{2} C_{n}^{n / 2}}{2 C_{n-1}^{n / 4-1}}
$$

частей меньшего диаметра. Однако размерность пространства, в котором лежит наш $(0,1)$-многогранник, равна $d=n(n-1) / 2$. Переписьвая полученную оценку в терминах величины $d$, получаем в точности то, что и требовалось: $f(d) \geqslant(1.203 \ldots+$ $o(1))^{\sqrt{d}}$.

Итак, мы видели, что оценка Кана и Калаи вписывается в рамки общего метода и что, более того, роль целочисленного многогранника здесь играет $(0,1)$-многогранник. Покажем теперь, как с помошью кросс-политопа удается слегка усилить доказанньй результат.

2. Доказательство оценки автора $f(d) \geqslant(2 / \sqrt{3}+o(1))^{\sqrt{2} \sqrt{d}}=(1.225 \ldots+$ $o(1))^{\sqrt{d}}$ (см. [44]). Если доказательство оценки Кана-Калаи было основано на применении теоремы Франкла-Вилсона, то для доказательства нашего результата мы будем пользоваться некоторым обобщением линейно-алгебраического метода Нилли (см. [40], [75] и [81]).

Пусть $n \in \mathbb{N}$ - достаточно большое натуральное число, $\delta=1-1 / \sqrt{3}$, а $p$ - ближайшее к величине $\delta n$ нечетное простое число. Известно (см. [85]), что для достаточно большого $x$ и для некоторого $\alpha<1$ (например, для $\alpha=38 / 61$ ) между $x$ и $x+x^{\alpha}$ лежит простое число. Поэтому можно считать, что $\delta n-(\delta n)^{\alpha}<p<\delta n+(\delta n)^{\alpha}$. Заметим, впрочем, что выбор величины $\alpha$ влияет исключительно на $o(1)$ из формулировки результата. Рассмотрим следующее семейство векторов $\Sigma \subset \mathbb{R}^{n}$ :

$$
\begin{aligned}
\Sigma=\left\{\mathbf{x}=\left(x_{1}, \ldots, x_{n}\right):\right. & x_{i} \in\{0,1,-1\} ; x_{i_{1}}=1, \\
& \left.x_{i_{2}}= \pm 1, \ldots, x_{i_{p}}= \pm 1,1 \leqslant i_{1}<\cdots<i_{p} \leqslant n\right\} .
\end{aligned}
$$

Иными словами, семейство векторов $\Sigma$ таково, что всякий вектор $\mathbf{x} \in \Sigma$ содержит в точности $n-p$ нулевых компонент и ровно $p$ компонент вида $x_{i_{1}}=1, x_{i_{2}}= \pm 1, \ldots, x_{i_{p}}$ $= \pm 1\left(1 \leqslant i_{1}<i_{2}<\cdots<i_{p} \leqslant n\right)$. Таким образом, $\Sigma$ есть определяющая система для некоторого кросс-политопа. Покажем, что на основе такого кросс-политопа можно получить требуемую оценку. В самом деле, ясно, что $\operatorname{card} \Sigma=2^{p-1} C_{n}^{p}$. Кроме того, нетрудно видеть, что для любых различных векторов $\mathbf{a}, \mathbf{b} \in \Sigma$ вьполнено следующее простое утверждение:

$$
(\mathbf{a}, \mathbf{b}) \equiv 0(\bmod p) \text { тогда и только тогда, когда }(\mathbf{a}, \mathbf{b})=0 .
$$

Каждому вектору $\mathbf{a} \in \Sigma$ поставим в соответствие многочлен $P_{\mathbf{a}} \in \mathbb{Z} / p \mathbb{Z}\left[x_{1}, \ldots, x_{n}\right]$, определяемый соотношениями $P_{\mathbf{a}}(\mathbf{x})=\prod_{i=1}^{p-1}(i-(\mathbf{a}, \mathbf{x}))$ и $x_{i}^{3}=x_{i}$. Тогда имеет место следующая 
Лемма. Каково бы ни было семейство векторов $Q=\left\{\mathbf{a}_{1}, \ldots, \mathbf{a}_{s}\right\} \subset \Sigma$ такое, что $\left(\mathbf{a}_{i}, \mathbf{a}_{j}\right) \not \equiv 0(\bmod p) \forall i \neq j$, для величины $s=\operatorname{card} Q$ выполнена оценка: ${ }^{5}$

$$
s \leqslant \sum_{l=0}^{p-1} \sum_{k=0}^{\left[\frac{p-1-l}{2}\right]} C_{n}^{k} C_{n-k}^{p-1-l-2 k}
$$

ДокАЗАТЕЛЬСТво. Для каждого вектора $\mathbf{a}_{i} \in Q$ рассмотрим отвечающий ему многочлен $P_{\mathbf{a}_{i}}$. Понятно тогда с учетом условия леммы и утверждения $(1)$, что многочлены $P_{\mathbf{a}_{1}}, \ldots, P_{\mathbf{a}_{s}}$ линейно независимы над $\mathbb{Z} / p \mathbb{Z}$. Но размерность пространства таких многочленов в силу их построения не превосходит как раз величины

$$
\sum_{l=0}^{p-1} \sum_{k=0}^{\left.\frac{[p-1-l}{2}\right]} C_{n}^{k} C_{n-k}^{p-1-l-2 k}
$$

и, стало быть, оценка (2), а вместе с ней и лемма доказаны полностью.

Заметим, что приведенное доказательство стандартно: аналогичные доказательства могут быть найдены в работах [40]-[45], а также в книгах [23], [46], [75].

Семейству векторов $\Sigma$ сопоставим семейство $n^{2}$-мерных векторов $\Sigma^{*}$ следуюшим образом. Каждому вектору $\mathbf{x} \in \Sigma$ поставим в соответствие вектор $\mathbf{x} * \mathbf{x}=\left(x_{i} \times x_{j}\right)$, $i=1, \ldots, n, j=1, \ldots, n$. Таким образом, $\Sigma^{*}$ тоже является определяющей системой точек для некоторого кросс-политопа. Легко убедиться в том, что для любых двух векторов $\mathbf{x} * \mathbf{x}, \mathbf{z} * \mathbf{z} \in \Sigma^{*}$ вьполнено равенство $(\mathbf{x} * \mathbf{x}, \mathbf{z} * \mathbf{z})=(\mathbf{x}, \mathbf{z})^{2}$. Значит, диаметр кросс-политопа с определяющей системой $\Sigma^{*}$ достигается на тех и только тех парах точек из $\Sigma^{*}$, скалярное произведение прообразов которых равно нулю. Воспользовавшись последним рассуждением, утверждением (1) и неравенством (2), получаем по принщипу Дирихле, что наш кросс-политоп невозможно разбить на $f<2^{p-1} C_{n}^{p} /\left(\sum_{l=0}^{p-1} \sum_{k=0}^{\left[\frac{p-1-l}{2}\right]} C_{n}^{k} C_{n-k}^{p-1-l-2 k}\right)$ частей так, чтобы диаметр каждой части был меньше диаметра всего кросс-политопа. Кроме того, понятно, что размерность этого кросс-политопа равна $\frac{n(n+1)}{2}-1$. Положим $d=\frac{n(n+1)}{2}-1$. Из сказанного выше следует, что $f(d) \geqslant 2^{p-1} C_{n}^{p} /\left(\sum_{l=0}^{p-1} \sum_{k=0}^{\left[\frac{p-1-l}{2}\right]} C_{n}^{k} C_{n-k}^{p-1-l-2 k}\right)$. Учитьвая выбор величины $p$ и проведя несложные выкладки, можно показать, что тем самым $f(d) \geqslant(2 / \sqrt{3}+o(1))^{\sqrt{2} \sqrt{d}}$ и наша оценка доказана.

ЗАмечАнИЕ 1. Итак, мы убедились в том, что, в самом деле, с помощью кросс-политопа удается получить оценку, лучшую чем та, что была ранее получена с помошью $(0,1)$-многогранника. Естественно было бы предположить, что возможны дальнейшие усиления имеюшихся оценок за счет рассмотрения некоторых более общих целочисленных многогранников. Тем не менее автору подобные примеры не известны, и представляется весьма вероятным, что для их построения потребуется разработка сушественно новых методов получения оценок вида (2).

\footnotetext{
${ }^{5}$ Суть леммы поясняется в точности так же, как это было сделано в замечании 1 из п. 1.
} 
ЗАмЕчАниЕ 2. В доказательстве оценки автора нет необходимости предполагать, что $p$ - простое число. Достаточно считать, что $p=q^{\alpha}$, где $q$ - простое, а $\alpha \geqslant 1$. При этом потребуется лишь внести некоторые изменения в определение многочленов $P_{\mathbf{a}}$ (см. [42] и [43]). Заметим также, что оценки вида (2) заведомо не всегда точны. Например, положим $n=8, p=4$, а

$$
\begin{aligned}
\Sigma=\{\mathbf{x}= & \left(x_{1}, \ldots, x_{n}\right): x_{i} \in\{0,1,-1\} ; x_{i_{1}}=1, \\
& \left.x_{i_{2}}= \pm 1, \ldots, x_{i_{p}}= \pm 1,1 \leqslant i_{1}<\cdots<i_{p} \leqslant n\right\} .
\end{aligned}
$$

Пусть $Q \subset \Sigma$ определено так же, как это было сделано в лемме. Тогда из элементарных соображений легко получить оценку $\operatorname{card} Q \leqslant 70$. В то же время линейно-алгебраический метод, с помошью которого была получена оценка (2), позволяет лишь утверждать, что card $Q \leqslant 157$. Все это подсказывает формулировку следуюшей гипотезы.

ГИПОТЕЗА. Пусть $n=2^{k}, q=2^{k-1}$, семейство векторов $\Sigma$ определено при этих $n$ и q так жее, как в (3), а семейство векторов $Q \subset \Sigma$ удовлетворяет стандартным условиям попарной неортогональности своих элементов. Тогда $\operatorname{card} Q \leqslant R(n)$, где, по крайней мере, $R(n) \leqslant 2^{n}$

Если гипотеза верна, то для $f(d)$ может быть получена оценка

$$
f(d) \geqslant(\sqrt{2}+o(1))^{\sqrt{2} \sqrt{d}}=(1.632 \ldots+o(1))^{\sqrt{d}} .
$$

Более того, очень вероятно тогда, что гипотезу Борсука удастся опровергнуть уже в размерности $d=135$.

Прежде чем переходить к получению оценок для хроматических чисел пространств, автору хотелось бы поблагодарить В. Судакова за указание некоторой неточности в первоначальной формулировке гипотезы (см. [86]).

3. Доказательство оценки Франкла и Вилсона $\chi\left(\mathbb{R}^{d}\right) \geqslant(1.207 \ldots+o(1))^{d}$. Как и доказательство оценки Кана-Калаи, предлагаемое доказательство также основано на применении теоремы Франкла-Вилсона.

Пусть $d$ - достаточно большое натуральное число, а $q=p^{\alpha}-$ некоторая степень простого числа $p$, причем $2 q \leqslant d$. Рассмотрим следуюшее семейство векторов $S \subset \mathbb{R}^{d}$ :

$$
\begin{aligned}
S=\{\mathbf{x}= & \left(x_{1}, \ldots, x_{d}\right): x_{i} \in\{0,1\} \\
& \left.x_{i_{1}}=1, \ldots, x_{i_{2 q-1}}=1,1 \leqslant i_{1}<\cdots<i_{2 q-1} \leqslant d\right\},
\end{aligned}
$$

т.е. каждый вектор $\mathbf{x} \in S$ имеет ровно $2 q-1$ координату, равную единице, и ровно $n-2 q+1$ координату, равную нулю. При этом ясно, что card $S=C_{d}^{2 q-1}$. Рассмотрим семейство векторов $S$ как $(M, D)$-критическую конфигурацию с критическим расстоянием $\sqrt{2 q}$ и с $M=C_{d}^{2 q-1}$. Понятно, с другой стороны, что $S$ можно интерпретировать как определяюшую систему для некоторого $(0,1)$-многогранника. Стало быть, и здесь мы оказываемся в рамках обшего метода, и для завершения доказательства требуемой оценки достаточно надлежашим образом оценить величину $D$ (здесь мы пользуемся тем фактом, что $\chi\left(\mathbb{R}^{d}\right)=\chi\left(\mathbb{R}^{d}, a\right)$ для любого вещественного числа $a>0$ (см. раздел 4.1)). Оценим $D$. С этой целью семейству векторов $S \subset \mathbb{R}^{d}$ сопоставим стандартным образом совокупность множеств $\mathscr{M} \subset 2^{\Re_{d}}$. Тогда критическое расстояние в конфигурации $S$ достигается на тех и только тех парах векторов из $S$, которым 
соответствуют множества $M_{1}, M_{2} \in \mathscr{M}$ с условием $\operatorname{card}\left(M_{1} \cap M_{2}\right)=q-1$. Однако в силу теоремы Франкла-Вилсона в любой подсовокупности $\mathscr{L} \subset \mathscr{M}$ такой, что card $\mathscr{L}>C_{d}^{q-1}$, найдется пара множеств $L_{1}, L_{2}$ с мошностью пересечения равной $q-1$. Значит, $D \leqslant C_{d}^{q-1}$. Проведенное рассуждение верно для всех $q$ с ограничением $2 q \leqslant d$, так что

$$
\chi\left(\mathbb{R}^{d}\right) \geqslant \max _{2 q \leqslant d, q=p^{\alpha}} \frac{C_{d}^{2 q-1}}{C_{d}^{q-1}} .
$$

Применяя асимптотический закон распределения простых чисел (см. [85]), получаем отсюда, что, в самом деле, $\chi\left(\mathbb{R}^{d}\right) \geqslant(1.207 \ldots+o(1))^{d}$ и оценка доказана полностью.

Итак, опять-таки полученная оценка вписьвается в рамки общего метода, и роль критической конфигурации здесь играет определяющая система для $(0,1)$-многогранника. В следуюшем пункте мы изложим недавний результат автора (см. [74]), позволяющий с помошью рассмотрения определяющей системы точек для некоторого кросс-политопа слегка улучшить оценку Франкла-Вилсона.

4. Доказательство теоремы 3 из раздела 3.3. Как и в п. 2 настоящего раздела, мы воспользуемся для доказательства некоторым обобщением линейно-алгебраического метода (см. [75] и [81]), которое было предложено автором в работах [44] и [74].

Пусть $x_{0}$ и $y_{0}$ такие же, как в формулировке теоремы. Пусть, далее, $d \in \mathbb{N}$ - достаточно велико, $a=\left[x_{0} d\right], b=\left[y_{0} d\right]$, а $p$ - наименшшее нечетное простое число, большее величины $[(a+2 b) / 2]$. Как и при доказательстве оценки в п. 2 текущего раздела, можно заметить со ссылкой на [85], что для достаточно большого $x$ и для некоторого $\alpha<1$ между $x$ и $x+x^{\alpha}$ лежит простое число и, стало быть, $p<[(a+2 b) / 2]+[(a+2 b) / 2]^{\alpha}$. Положим

$$
\begin{aligned}
\Sigma=\left\{\mathbf{x}=\left(x_{1}, \ldots, x_{d}\right): x_{i} \in\{0,1,-1\}\right. & \\
& \left.\operatorname{card}\left\{x_{i}= \pm 1\right\}=a ; \operatorname{card}\left\{x_{i}=-1\right\}=b\right\} \subset \mathbb{R}^{d} .
\end{aligned}
$$

Понятно тогда, что $\Sigma$ является определяющей системой точек для некоторого кроссполитопа и что, более того, $M=\operatorname{card} \Sigma=C_{d}^{a} C_{a}^{b}$. Нетрудно видеть теперь, что имеет место следуюшее простое утверждение, аналогичное утверждению (1) из п. 2 :

$$
\begin{gathered}
\forall \mathbf{x}, \mathbf{y} \in \Sigma(\mathbf{x} \neq \mathbf{y}) \text { выполнено } \\
(\mathbf{x}, \mathbf{y}) \equiv a(\bmod p) \text { тогда и только тогда, когда }(\mathbf{x}, \mathbf{y})=a-p .
\end{gathered}
$$

Как и в п. 2, каждому вектору $\mathbf{x} \in \Sigma$ поставим в соответствие многочлен $P_{\mathbf{x}} \in$ $\mathbb{Z} / p \mathbb{Z}\left[x_{1}, \ldots, x_{d}\right]$, определяемьй соотношениями $P_{\mathbf{x}}(\mathbf{y})=\prod_{i \neq a(\bmod p)}(i-(\mathbf{x}, \mathbf{y})), x_{i}^{3}=$ $x_{i}, i=1, \ldots, d$. Рассмотрим, далее, произвольное множество $Q=\left\{\mathbf{x}_{1}, \ldots, \mathbf{x}_{s}\right\} \subset \Sigma$ такое, что $\left(\mathbf{x}_{i}, \mathbf{x}_{j}\right) \not \equiv a(\bmod p) \forall i \neq j$. В этом случае также можно показать, что многочлены $P_{\mathbf{x}_{1}}, \ldots, P_{\mathbf{x}_{s}}$ линейно независимы над полем $\mathbb{Z} / p \mathbb{Z}$, а значит,

$$
s \leqslant \sum_{i=0}^{p-1} \sum_{j=0}^{[(p-1-i) / 2]} C_{d}^{j} C_{d-j}^{p-1-i-2 j}=D .
$$

Из утверждения (4) и неравенства (5) немедленно следует, что определяющая система $\Sigma$ является одновременно и $(M, D)$-критической конфигурацией с критическим 
расстоянием $\sqrt{2 p}$ и величинами $M$ и $D$, определенными в ходе доказательства. Учитьвая выбор параметров в формулировке и доказательстве теоремы и произведя несложные выкладки, получаем:

$$
\chi\left(\mathbb{R}^{d}\right) \geqslant M / D \geqslant(\gamma+o(1))^{d}=(1.239 \ldots+o(1))^{d} .
$$

Теорема доказана.

ЗАмечАнИЕ 1. Поясним выбор параметров в формулировке только что доказанной теоремы. Для этого заметим, что в проведенном доказательстве величины $a$ и $b$ можно считать изначально выбранными произвольно с условием $a \leqslant d, b \leqslant d, a \asymp d, b \asymp d$. В самом деле, соответствуюшее семейство векторов $\Sigma$ все равно можно будет интерпретировать и как определяющую систему для кросс-политопа, и как $(M, D)$-критическую конфигурацию, так что, как и прежде, $\chi\left(\mathbb{R}^{d}\right) \geqslant M / D$. При этом $M=M(a, b)=C_{d}^{a} C_{a}^{b}$, $D=D(a, b)=\sum_{i=0}^{p-1} \sum_{j=0}^{[(p-1-i) / 2]} C_{d}^{j} C_{d-j}^{p-1-i-2 j}$ (здесь $D=D(a, b)$, поскольку $p=p(a, b))$. Но тогда ясно, что $\chi\left(\mathbb{R}^{d}\right) \geqslant \max _{a, b} M(a, b) / D(a, b)$, и мы приходим к несложной вариационной задаче. Для решения этой задачи достаточно сперва слегка упростить выражение для $D(a, b)$, заменив двойную сумму максимальным слагаемым и получив тем самым оценку $\chi\left(\mathbb{R}^{d}\right) \geqslant P^{-1}(d) \max _{a, b} M(a, b) / D^{\prime}(a, b)$, где $P(d)-$ некоторьй полином. Затем следует выписать необходимые условия экстремума и убедиться в том, что они же, в действительности, являются и достаточньми. Как известно, необходимые условия имеют вид:

$$
\frac{\partial}{\partial a}\left(\frac{M(a, b)}{D^{\prime}(a, b)}\right)=0 ; \quad \frac{\partial}{\partial b}\left(\frac{M(a, b)}{D^{\prime}(a, b)}\right)=0 .
$$

Наконец, расписав с учетом формулы Стирлинга в явном виде выражения для частных производных, нетрудно убедиться в том, что система (6) эквивалентна системе $(*),(* *)$ из формулировки теоремы (разумеется, всюду здесь следует предполагать, что $a=a^{\prime} d, b=b^{\prime} d, 0.3 \leqslant a^{\prime} \leqslant 0.4,0.01 \leqslant b^{\prime} \leqslant 0.1$, и пренебрегать несушественными сомножителями вида $\sqrt{2 \pi x}$ ). Поскольку, как мы уже замечали, условия (6) являются не только необходимыми, но и достаточными, параметры в формулировке теоремы подобраны оптимальным образом.

ЗАмечАниЕ 2. Все рассуждения из замечания 1 имеют смысл лишш постольку, поскольку мы не знаем, верна ли гипотеза из п. 2 настоящего раздела. Однако если эта гипотеза все же верна, то для $\chi\left(\mathbb{R}^{d}\right)$ может быть получена оценка $\chi\left(\mathbb{R}^{d}\right) \geqslant(\sqrt{2}+o(1))^{d}$.

ЗАмечАниЕ 3. Наконец, следует отметить, что автору не известны примеры нетривиальных определяюших систем для целочисленных многогранников более общего вида, чем $(0,1)$-многогранник и кросс-политоп, которые бы позволяли получать оценки для хроматического числа вешественного пространства лучшие, чем те, что были получены в последних двух пунктах текушего раздела.

Итак, мы доказали асимптотические нижние оценки хроматического числа вешественного пространства, сформулированные в $§ 3$. В следующем пункте естественно перейти к доказательству аналогичных оценок для рационального случая. Однако ввиду близости подходов к доказательству соответствующих оценок мы не станем проводить доказательство оценки Франкла-Вилсона $\chi\left(\mathbb{Q}^{d}\right) \geqslant(1.139 \ldots+o(1))^{d}$, заметив 
только, что оно может быть получено в рамках общего метода с помошью построения определяющей системы точек для некоторого $(0,1)$-многогранника и что схожие результаты могут быть найдены в книге [75]. Перейдем, стало быть, непосредственно к доказательству недавней оценки автора $\chi\left(\mathbb{Q}^{d}\right) \geqslant(1.173 \ldots+o(1))^{d}($ см. теорему 4 из раздела 3.3$)$.

5. Доказательство теоремы 4 из раздела 3.3. Предлагаемое доказательство достаточно близко к доказательству из предыдущего пункта, однако здесь имеется и ряд весьма существенных отличий.

Пусть $d$ - достаточно большое натуральное число. Выберем натуральное число $k$ из условия $2^{2 k} \leqslant d<2^{2 k+2}$ (ясно, что тем самьм $k$ определено однозначно). Положим $q=2^{2 k-1}, l=q / n$, так что $\frac{1}{8}<l \leqslant \frac{1}{2}$ и введенные обозначения коррелируют с обозначениями из формулировки теоремы 4 . Таким образом, мы можем рассмотреть величину $y_{l}$ из той же формулировки и величину $x_{l}=2 l-2 y_{l}$. Определим $a=\left[x_{l} d\right], b=\left[y_{l} d\right]-1$ и построим семейство векторов $\Sigma \subset \mathbb{Q}^{d}$ в точности так же, как было построено одноименное семейство векторов из предыдушего пункта:

$$
\begin{aligned}
\Sigma=\{\mathbf{x}= & \left(x_{1}, \ldots, x_{d}\right): x_{i} \in\{0,1,-1\} ; \\
& \left.\operatorname{card}\left\{x_{i}= \pm 1\right\}=a ; \operatorname{card}\left\{x_{i}=-1\right\}=b\right\} \subset \mathbb{Q}^{d} .
\end{aligned}
$$

Понятно тогда, что и здесь мы имеем дело с определяющей системой точек для некоторого кросс-политопа; только на сей раз нам будет необходимо проинтерпретировать эту систему как $(M, D)$-критическую конфигурацию не в $\mathbb{R}^{d}$, а в $\mathbb{Q}^{d}$. Это означает, что критическое расстояние обязано быть рациональньм числом (в то время как в п. 4 текущего раздела оно равнялось иррациональной величине $\sqrt{2 p}$, где $p$ - нечетное простое число). Покажем, что, действительно, параметры подобраны таким образом, что подобная интерпретация возможна и обший метод работает и здесь.

В самом деле, рассмотрим $M=C_{d}^{a} C_{a}^{b}$, а величину $D$ определим в соответствии со стандартной леммой:

Лемма. Каково бы ни было семейство векторов $Q=\left\{\mathbf{x}_{1}, \ldots, \mathbf{x}_{s}\right\} \subset \Sigma$ с условием $\left(\mathbf{x}_{i}, \mathbf{x}_{j}\right) \not \equiv a(\bmod q) \forall i \neq j$, имеет место оиенка

$$
s \leqslant \sum_{i=0}^{q-1} \sum_{j=0}^{[(q-1-i) / 2]} C_{d}^{j} C_{d-j}^{q-1-i-2 j}=D .
$$

Доказательство этой леммы опять-таки основывается на том, что каждому вектору $\mathbf{x}$ из $\Sigma$ мы сопоставляем некоторый многочлен $P_{\mathbf{x}}$ и доказываем линейную независимость многочленов, отвечаюших элементам из $Q$. Тем не менее существенное отличие состоит в том, что на сей раз мы вынуждены рассматривать такие многочлены как элементы из $\mathbb{Q}\left[x_{1}, \ldots, x_{d}\right]$ и линейную независимость понимать не как независимость над полем классов вычетов по простому модулю, а как независимость над полем рациональных чисел. Все это создает дополнительные технические трудности, однако способ борьбы с этими трудностями сушествует и описан он в работах [42] и [43]. Посему доказательство леммы мы в настоящей работе также не приводим, заметив только, что упомянутые многочлены могут быть получены из соотношений

$$
P_{\mathbf{x}}(\mathbf{y})=\frac{1}{2^{T}} \prod_{i \neq a(\bmod q)}(i-(\mathbf{x}, \mathbf{y})), \quad x_{i}^{3}=x_{i}, \quad i=1, \ldots, d,
$$

где $T$ таково, что $2^{T} \mid \prod_{i \not \equiv a(\bmod q)} i$, a $2^{T+1} \nmid \prod_{i \neq a(\bmod q)} i$. 
Исходя из очевидного неравенства $a+2 b<2 q$, можно доказать теперь утверждение, аналогичное утверждениям (1) и (4). Тогда, с учетом этого утверждения и леммы мы получаем, что $\Sigma$ есть $(M, D)$-критическая конфигурация с критическим расстоянием, равным величине $\sqrt{2 q}=2^{k} \in \mathbb{Z}$. Таким образом, для завершения оценки остается заметить, что $M=M(l), D=D(l)$ и априори мы не можем сказать, чему равна величина $l$ из полуинтервала $\left(\frac{1}{8}, \frac{1}{2}\right]$, так что, вспоминая выбор параметров и производя несложные выкладки, мы как раз и получаем:

$$
\chi\left(\mathbb{Q}^{d}\right) \geqslant \min _{l} M(l) / D(l) \geqslant\left(\min _{l} \gamma_{l}+o(1)\right)^{d}=(1.173 \ldots+o(1))^{d} .
$$

Теорема 4 доказана.

ЗАмечАнИЕ. Отметим сперва, что, как и прежде, с помошюю существующих методов не удается, по-видимому, получать новые оценки для $\chi\left(\mathbb{Q}^{d}\right)$ за счет рассмотрения целочисленных многогранников более общего вида, чем $(0,1)$-многогранник или кросс-политоп. Отметим, далее, что, как и в теореме 3 , параметры в формулировке и доказательстве теоремы 4 подобраны оптимальным образом (см. замечание 1 из п. 4). Однако и здесь такой выбор имеет смысл лишш до тех пор, пока мы не убедились в вероятной справедливости гипотезы. Отметим, наконец, что по некоторой последовательности $d_{k} \rightarrow \infty($ при $k \rightarrow \infty)$ для величины $\chi\left(\mathbb{Q}^{d_{k}}\right)$ может быть получена в точности та же оценка, что и для величины $\chi\left(\mathbb{R}^{d_{k}}\right)$, и, следовательно, суть теоремы 4 как раз в том и состоит, что с ее помощью удается получать нетривиальные оценки в произвольной размерности $d$, а не только в некотором (хотя бы и бесконечном) множестве ее значений.

До сих пор мы нигде не использовали целочисленные многогранники более общего вида, чем $(0,1)$-многогранник или кросс-политоп; более того, мы неоднократно отмечали, что наш подход не позволяет улучшать те или иные уже полученные оценки с помощю таких целочисленных многогранников (см. замечание 1 в п. 2 настоящего раздела, замечание 3 в п. 4 и замечание в п. 5). В результате может даже возникнуть вопрос: а нужны ли вообще эти многогранники? Положительньй ответ на этот вопрос будет дан в следующем пункте, где будет доказана недавняя теорема автора, сформулированная в разделе 3.4 .

6. Доказательство теоремы из раздела 3.4. Сперва заметим, что доказательство оценки $\chi\left(\mathbb{R}^{d}, a_{1}, a_{2}\right) \geqslant(1.439 \ldots+o(1))^{d}$ с некоторьми $a_{1}>0, a_{2}>0$ практически дословно повторяет доказательство теоремы 3 из раздела 3.3 , которое было проведено нами в п. 4. Разнища состоит лишш в выборе параметров, а также в том, что здесь мы снова построим некоторую определяющую систему точек для кросс-политопа, однако на сей раз мы будем интерпретировать ее не как $(M, D)$-критическую конфигурацию, а как $(M, D, 2)$-критическую конфигурацию с запрешенными расстояниями $a_{1}$ и $a_{2}$. Таким образом, и тут мы оказьваемся в рамках общего метода, и для иллюстрации доказательства нам достаточно вьписать необходимые параметры и сослаться на п. 4. Параметры же таковы: $d$ - достаточно большое натуральное число, $s=\left[x_{0} d\right], t=\left[y_{0} d\right]\left(x_{0}, y_{0}\right.$ - величины, определенные в формулировке теоремы), $p-$ наименьшее нечетное простое число, строго большее величины $[(s+2 t) / 3]$, а семейство векторов $\Sigma$ (определяюшая система для кросс-политопа) имеет формально тот же 
вид, что и одноименное семейство векторов из п. 4 :

$$
\begin{aligned}
\Sigma=\{\mathbf{x}= & \left(x_{1}, \ldots, x_{d}\right): x_{i} \in\{0,1,-1\} \\
& \left.\quad \operatorname{card}\left\{x_{i}= \pm 1\right\}=s ; \operatorname{card}\left\{x_{i}=-1\right\}=t\right\} \subset \mathbb{R}^{d} .
\end{aligned}
$$

Наконец, $M=C_{d}^{s} C_{s}^{t}, D=\sum_{i=0}^{p-1} \sum_{j=0}^{[(p-1-i) / 2]} C_{d}^{j} C_{d-j}^{p-1-i-2 j}$, а запрещенные расстояния $a_{1}, a_{2}$ отвечают тем парам векторов $x, y \in \Sigma$, скалярное произведение которых равно либо $s-p$, либо $s-2 p$. Произведя все необходимые рассуждения и выкладки, нетрудно проверить, что тогда, в самом деле, $\chi\left(\mathbb{R}^{d}\right) \geqslant(\gamma+o(1))^{d}=(1.439 \ldots+o(1))^{d}$.

Заметим, что наилучшая оценка, которую здесь удается получить с помошью определяющих систем точек для $(0,1)$-многогранников, имеет вид $\chi\left(\mathbb{R}^{d}, a_{1}, a_{2}\right) \geqslant$ $\left(\frac{4}{3}+o(1)\right)^{d}$, так что рассмотрение определяющих систем для кросс-политопов и в этом случае оказьвается более эффективным. Заметим, кстати, что и здесь все параметры подобраны оптимально (см. замечание 1 в п. 4 настолшего раздела).

Перейдем теперь к доказательству основного результата теоремы: существуют такие абсолютные константы $c_{1}>0, c_{2}>0$, что $\chi\left(\mathbb{R}^{d}, a_{1}, \ldots, a_{k}\right) \geqslant\left(c_{1} k\right)^{c_{2} d}$ при некоторых значениях величин $a_{1}>0, \ldots, a_{k}>0$. Зафиксируем достаточно большое значение размерности $d>d_{0}$ (в дальнейшем будет видно, насколько большой может оказаться абсолютная постоянная $\left.d_{0}\right)$ и рассмотрим четыре случая.

Случай 1$)$. Пусть $k \leqslant 2^{10000}$. Тогда из очевидного неравенства $\chi\left(\mathbb{R}^{d}, a_{1}, \ldots, a_{k}\right) \geqslant$ $\chi\left(\mathbb{R}^{d}\right)$, верного для любого набора неотрицательных вещественных чисел $a_{1}, \ldots, a_{k}$, немедленно следует оценка $\chi\left(\mathbb{R}^{d}, a_{1}, \ldots, a_{k}\right) \geqslant(1.2)^{d}=\left(\nu_{1} k\right)^{\mu_{1} d}$ (здесь при написании последнего равенства мы пользуемся изначальньм предположением о том, что $k$ не превосходит некоторой константы). Стало быть, в этом случае требуемая оценка доказана, причем для ее получения достаточно было использовать $(M, D)$-критическую конфигурацию, построенную даже на основе определяющей системы точек для $(0,1)$-многогранника.

Случай 2). Пусть теперь $2^{10000}<k \leqslant 2^{100} d^{2}\left(d>d_{0}\right)$. В этом случае нам потребуется ввести некоторые дополнительные параметры. Положим $r=\left[\frac{1}{2} \log _{2} k\right]-100$, $t=\left[d / 2^{r}\right]$. Ясно, что тогда $r \leqslant\left[\log _{2} d\right]$ и, значит, $t \geqslant 1$. Рассмотрим множество $\Re_{2^{r} t}=\left\{1, \ldots, 2^{r} t\right\} \subset \Re_{d}$ и разобьем его на произвольные $2^{r}$ частей мошности $t$ :

$$
\Re_{2^{r} t}=A_{1} \sqcup \cdots \sqcup A_{2^{r}}, \quad \operatorname{card} A_{i}=t .
$$

Каждому такому разбиению поставим в соответствие вектор $\mathbf{x}=\mathbf{x}\left(A_{1}, \ldots, A_{2^{r}}\right) \in \mathbb{R}^{d}$ следующим образом:

$$
\begin{aligned}
\mathbf{x}\left(A_{1}, \ldots, A_{2^{r}}\right)=\left(x_{1}, \ldots, x_{d}\right):\left\{i: x_{i}=1\right\}=A_{1} ;\left\{i: x_{i}=2\right\}=A_{2} ; \ldots ; \\
\\
\left\{i: x_{i}=2^{r}\right\}=A_{2^{r}} ;\left\{i: x_{i}=0\right\}=\Re_{d} \backslash \Re_{2^{r}} .
\end{aligned}
$$

Наконец, положим $\Sigma=\bigcup \mathbf{x}\left(A_{1}, \ldots, A_{2^{r}}\right)$, где объединение берется по всем возможным разбиениям множества $\Re_{2^{r} t}$ на $2^{r}$ частей мошности $t$, причем разбиения считаются различными, даже если все их элементы в совокупности одинаковы, но записаны в разном порядке: например, $\left\{A, B, A_{3}, \ldots, A_{2^{r}}\right\} \neq\left\{B, A, A_{3}, \ldots, A_{2^{r}}\right\}$. Тем самым мы имеем определяющую систему точек для некоторого целочисленного многогранника весьма общего вида. Нашей дальнейшей целью будет доказательство того, что 
такая определяющая система является одновременно и $(M, D, k)$-критической конфигурацией с $M / D \geqslant\left(\nu_{2} k\right)^{\mu_{2} d}\left(\nu_{2}, \mu_{2}\right.$ - некоторые абсолютные постоянные) и что, стало быть, общий метод вновь оказьвается применимым.

В самом деле, зададим величину $M$ равенством $M=\operatorname{card} \Sigma$. Тогда нетрудно показать, что $M \geqslant 2^{r d / 2-2 d}$. Положим

$$
a=\left[\frac{d}{2^{r}}\right] \frac{2^{r}\left(2^{r}+1\right)\left(2^{r+1}+1\right)}{6}
$$

(заметим, что на самом деле $a=(\mathbf{x}, \mathbf{x})$ для любого вектора $\mathbf{x} \in \Sigma$ ) и рассмотрим наименьшее нечетное простое число $p>a / k$. Тогда в силу известного постулата Бертрана $p \leqslant 2 a / k$ (здесь мы снова пользуемся тем, что $d>d_{0}$, для обоснования неравенства $a / k>3)$. Определим, далее, величины $b_{1}=a-p, b_{2}=a-2 p, \ldots, b_{k}=a-k p$ и каждому вектору $\mathbf{x} \in \Sigma$ поставим в соответствие многочлен $P_{\mathbf{x}} \in \mathbb{Z} / p \mathbb{Z}\left[x_{1}, \ldots, x_{d}\right]$ следуюшего вида:

$$
P_{\mathbf{x}}(\mathbf{y})=\prod_{i \neq a(\bmod p)}(i-(\mathbf{x}, \mathbf{y})), \quad x_{i} \times\left(x_{i}-1\right) \times \cdots \times\left(x_{i}-2^{r}\right)=0 .
$$

Значит, каждьй такой многочлен имеет степень $p-1$, и любая переменная входит в этот многочлен в степени, не превосходящей величины $2^{r}$. Имеет место

Лемма. Каково бы ни было семейство векторов $Q=\left\{\mathbf{x}_{1}, \ldots, \mathbf{x}_{s}\right\} \subset \Sigma$ с условием $\left(\mathbf{x}_{i}, \mathbf{x}_{j}\right) \notin\left\{b_{1}, \ldots, b_{k}\right\}$, для величины $s=\operatorname{card} Q$ может быть получена оченка

$$
s \leqslant \sum_{l=0}^{p} C_{d}^{l} 2^{r l}<2^{r p+d}=D .
$$

Доказательство этой леммы стандартно (достаточно показать линейную независимость многочленов $P_{\mathbf{x}_{1}}, \ldots, P_{\mathbf{x}_{s}}$ над полем $\left.\mathbb{Z} / p \mathbb{Z}\right)$, и мы его здесь не приводим. Заметим только, что для получения окончательной оценки мы воспользовались также тем, что $p \leqslant 2 a / k<d$ (при $\left.d>d_{0}\right)$.

В свою очередь, из леммы следует, что, действительно, $\Sigma$ есть $(M, D, k)$-критическая конфигурация с величинами $M$ и $D$, определенными в ходе доказательства, и запрешенными расстояниями $a_{1}, \ldots, a_{k}$, отвечаюшими скалярным произведениям, равным $b_{1}, \ldots, b_{k}$. Стало быть, и в этом случае метод применим, и мы имеем оценку

$$
\chi\left(\mathbb{R}^{d}, a_{1}, \ldots, a_{k}\right) \geqslant M / D=2^{r d / 2-2 d-r p-d} \geqslant 2^{r d / 3}=\left(\nu_{2} k\right)^{\mu_{2} d} .
$$

Случай 3). В этом случае мы предполагаем, что

$$
\max \left\{2^{10000}, 2^{100} d^{2}\right\}<k \leqslant d^{2.5} \quad\left(d>d_{0}\right) .
$$

Тогда мы попросту "продолжаем" оценку из предыдущего пункта на такие значения $k$ : $\max \chi\left(\mathbb{R}^{d}, a_{1}, \ldots, a_{k}\right) \geqslant \max \chi\left(\mathbb{R}^{d}, a_{1}, \ldots, a_{l}\right)$ при $l<k$ (например, $\left.l=2^{100} d^{2}\right)$, и, следовательно, найдутся такие $a_{1}, \ldots, a_{k}$, что $\chi\left(\mathbb{R}^{d}, a_{1}, \ldots, a_{k}\right) \geqslant\left(\nu_{3} k\right)^{\mu_{3} d}$ (здесь мы пользуемся тем, что $k \leqslant d^{\alpha}$ с фиксированным $\left.\alpha\right)$. 
Случай 4). Рассмотрим, наконец, последний оставшийся случай - случай, когда $k>d^{2.5}$. Введем следующие обозначения: через $\mathbb{Z}_{+}^{d}$ мы обозначим множество всех векторов целочисленной решетки, имеюших неотрицательные координаты; через $B$ мы обозначим шар радиуса $\sqrt{k / 2}$ с центром в начале координат. Определим множество $S \subset \mathbb{R}^{d}$ как определяющую систему точек для целочисленного многогранника, получаемую в результате пересечения шара $B$ и множества $\mathbb{Z}_{+}^{d}$. Ясно тогда, что $S-$ это также и $(M, D, k)$-критическая конфигурация с $M=\operatorname{card} S \geqslant V_{d}(\gamma \sqrt{k})^{d}$ (где $V_{d}-$ объем единичного $d$-мерного шара, а $\gamma$ - абсолютная постоянная) и $D=1$. В самом деле,

$$
\operatorname{card}\left\{a_{i}>0: a_{i}=d(\mathbf{x}, \mathbf{y}), \mathbf{x}, \mathbf{y} \in S\right\} \leqslant k .
$$

Это немедленно следует из целочисленности квадратов расстояний между любыми векторами из $S$, неотрицательности всех координат каждого из таких векторов и того факта, что всякий вектор из $S$ принадлежит, в частности, шару радиуса $\sqrt{k / 2}$. Стало быть, в качестве множества запрешенных расстояний мы можем рассмотреть множество $\left\{a_{1}, \ldots, a_{l}\right\}=\left\{a_{i}>0: a_{i}=d(\mathbf{x}, \mathbf{y}), \mathbf{x}, \mathbf{y} \in S\right\}, l \leqslant k$, т.е., иными словами, множество всех различных расстояний между парами точек из $S$.

Итак, мы получили оценку $\chi\left(\mathbb{R}^{d}, a_{1}, \ldots, a_{k}\right) \geqslant M \geqslant\left(\nu_{4} k\right)^{\mu_{4} d}$ (здесь в последнем неравенстве мы воспользовались тем, что $\sqrt{k} / d \gg k^{1 / 10}$ и, значит, величина $V_{d}$ не влияет на вид требуемой оценки, а влияет лишш на значения констант).

Для завершения доказательства остается лишш положить $c_{1}=\min \left\{\nu_{1}, \ldots, \nu_{4}\right\}$, $c_{2}=\min \left\{\mu_{1}, \ldots, \mu_{4}\right\}$, так что всегда $\chi\left(\mathbb{R}^{d}, a_{1}, \ldots, a_{k}\right) \geqslant\left(c_{1} k\right)^{c_{2} d}$ при некоторых $a_{1}>0, \ldots, a_{k}>0$. Теорема доказана.

ЗАмечАнИЕ 1. В приведенном доказательстве мы намеренно не следили за значениями констант. Разумеется, можно было осуществить гораздо более аккуратньй счет; однако в том, по-видимому, нет никакого смысла: гораздо интереснее попытаться получить качественно лучшие оценки (см. замечание в разделе 3.4 ).

ЗАмечАние 2. В заключение стоит отметить, что разработанньй в данном разделе обший метод позволяет также получать очень хорошие (наилучшие из известных) нижние оценки и для хроматических чисел некоторых других метрических пространств. Например, с помошью этого метода удается весьма эффективно работать с пространствами вида $\mathbb{Q}^{d}\left(\alpha_{1}, \ldots, \alpha_{s}\right)=\mathbb{Q}^{d}(\alpha)$, т.е. с пространствами, которые в малых размерностях были изучены, в частности, в уже цитированных ранее работах [51] и [64] (ср. замечание из п. 2 в разделе 4.1 и замечание 1 из п. 3 того же раздела). Тем не менее получаемые таким образом оценки по существу мало отличаются от оценок для $\chi\left(\mathbb{Q}^{d}\right)$, и потому в настоящей работе мы их не приводим.

На этом следует завершить основную часть статьи. Тем не менее в следуюшем параграфе мы обсудим еше одну смежную задачу, которая естественным образом возникает в рамках изученной нами проблематики.

\section{§. Дополнение}

5.1. Проблема Борсука для $(0,1)$-многогранников и кросс-политопов. Итак, в предыдушем параграфе мы сформулировали обший метод, позволяюший эффективно работать как с проблемой Борсука, так и с задачей определения хромати- 
ческих чисел различных метрических пространств. Мы видели, что большинство результатов, описанных и доказанных в этом параграфе, получается с помошю рассмотрения некоторых $(0,1)$-многогранников и кросс-политопов. Возникает естественный вопрос: что можно сказать про минимальное число частей меньшего диаметра, если мы ограничимся рассмотрением только множеств указанного типа? Конечно, в этом случае гипотеза Борсука не может быть доказана (по крайней мере при $d \geqslant 560$ ), однако, во-первых, интересно понять, что будет в “малых" размерностях, а во-вторых, интересно попытаться с ростом размерности получить какие-нибудь верхние оценки, лучшие, чем те, что были получены для общей ситуации в работах [28] и [31]. Эти задачи мы и обсудим в настояшем параграфе.

Случай “малых" размерностей рассматривался только для $(0,1)$-многогранников (кросс-политопы не изучались) многими авторами, в числе которых можно назвать Ф. Шиллера (см. [87]), А. Схрейвера, Пайана (см. [88]), Й. Петерсена (см. [89]) и Г. М. Циглера (см. [90] и [91]). В результате к 1998 году гипотеза Борсука для $(0,1)-$ многогранников была доказана при всех $d \leqslant 9$. Иными словами, усилиями перечисленных вьше авторов был получен следуюший результат: при любом $d \leqslant 9$ каждое множество $\Omega \subset\{0,1\}^{d}$ может быть разбито на $d+1$ часть меньшего диаметра. Этот результат был основан на применении раскрасок хэмминговых графов и свойств оптимальных двоичных кодов. Подробности могут быть найдены в работе [91], и мы на них останавливаться не будем.

Многомерный случай - случай растущей размерности - был изучен (как для $(0,1)$ многогранников, так и для кросс-политопов) в 2000году автором в работе [92]. Чтобы описать результаты этой работы, необходимо дать сперва некоторые определения.

Через $\mathscr{F}_{k, l}^{d}=\left\{\mathbf{v}_{1}, \ldots, \mathbf{v}_{s}\right\} \subset \mathbb{R}^{d}$ мы будем обозначать произвольное семейство $(0,1)$-векторов такое, что $\left(\operatorname{diam} \mathscr{F}_{k, l}^{d}\right)^{2}=k, \mathrm{a}_{i}=\left(v_{i}^{1}, \ldots, v_{i}^{d}\right)$ и $v_{i}^{1}+\cdots+v_{i}^{d}=l \forall i$. Иными словами, семейство векторов $\mathscr{F}_{k, l}^{d}$ - это такое подмножество вершин гиперкуба $[0,1]^{d}$ (определяющая система для $(0,1)$-многогранника), что квадрат максимального расстояния между векторами, принадлежашими ему, равен $k$, а число ненулевых координат в каждом из векторов $\mathbf{v} \in \mathscr{F}_{k, l}^{d}$ совпадает с величиной $l$. Понятно тогда, что $k$ четно, $0 \leqslant k \leqslant d, 0 \leqslant l \leqslant d$ и, кроме того, $k \leqslant 2 l$, если $2 l \leqslant d$, и $k \leqslant 2 d-2 l$, если $2 l>d$. Определим теперь $\mathscr{F}_{k, l_{1}, l_{2}}^{d}=\left\{\mathbf{w}_{1}, \ldots, \mathbf{w}_{t}\right\} \subset \mathbb{R}^{d}$ как произвольное семейство $(0,1,-1)$-векторов такое, что $\left(\operatorname{diam} \mathscr{F}_{k, l_{1}, l_{2}}^{d}\right)^{2}=k, \mathrm{a} \mathrm{w}_{i}=\left(w_{i}^{1}, \ldots, w_{i}^{d}\right)$, $\left(w_{i}^{1}\right)^{2}+\cdots+\left(w_{i}^{d}\right)^{2}=l_{1}+l_{2}$ и $w_{i}^{1}+\cdots+w_{i}^{d}=l_{1}-l_{2} \forall i$. Иначе говоря, $\mathscr{F}_{k, l_{1}, l_{2}}^{d}-$ это такое подмножество множества вершин и центров граней промежуточной размерности гиперкуба $[-1,1]^{d}$ (определяющая система для кросс-политопа), что квадрат максимального расстояния между векторами, принадлежашими ему, равен $k$, число единичных координат в каждом из векторов $\mathbf{w} \in \mathscr{F}_{k, l_{1}, l_{2}}^{d}$ совпадает с величиной $l_{1}$, а число отрицательных компонент любого такого вектора равно $l_{2}$. Понятно, что и в этом определении нетрудно найти естественные ограничения на параметры $k, l_{1}$ и $l_{2}$.

Определим величину $f_{k, l}(d)$ как минимальное число такое, что всякое семейство векторов $\mathscr{F}_{k, l}^{d}$ разбивается на $f_{k, l}(d)$ частей меньшего диаметра. Рассмотрим также $f_{k, l_{1}, l_{2}}(d)$ - минимальное число такое, что всякое семейство векторов $\mathscr{F}_{k, l_{1}, l_{2}}^{d}$ разбивается на $f_{k, l_{1}, l_{2}}(d)$ частей меньшего диаметра.

В своей статье (см. [92]) автор приводит различные верхние оценки на величины $f_{k, l}(d)$ и $f_{k, l_{1}, l_{2}}(d)$. Оказывается, что для весьма широкого класса случаев (т.е. для 
многих различных значений величин $k, l, l_{1}$ и $l_{2}$ ) эти оценки лучше, чем общие верхние оценки на величину $f(d)$, полученные ранее в работах [28] и [31] Шраммом и Бургейном и Линденштрауссом. Точные формулировки соответствуюших теорем достаточно громоздки, и в настоящей работе мы их не приводим. Однако, дабы проиллюстрировать сказанное, мы все же приведем здесь несколько примеров, из которых видно, на наш взгляд, соотношение между результатами работ [92] и [28] ([31]).

Примеры. 1) Пусть $d=12 d^{\prime}, k=d / 6$, a $l=d / 3$. В этом случае в работе [92] получается оценка: $f_{k, l}(d) \leqslant P_{1}(d)(1.206 \ldots)^{d}$, где $P_{1}(d)$ - полином. Значит, в этом случае оценка из [92] лучше, чем оценка $f_{k, l}(d) \leqslant P(d)(3 / 2)^{d / 2}=P(d)(1.224 \ldots)^{d}$ $(P(d)$ - полином $)$, полученная в [28] и в [31].

2) Пусть $d=12 d^{\prime}, k=d / 2$, a $l=d / 3$. Тогда из результатов работы [92] вытекает оценка: $f_{k, l}(d) \leqslant P_{2}(d)(1.104 \ldots)^{d}$. Иными словами, здесь оценка снова лучше, чем в $[28]$ и в $[31]$.

3) Пусть $d=32 d^{\prime}, k=d / 4$, a $l_{1}=l_{2}=d / 16$. Тогда в силу [92] имеет место неравенство: $f_{k, l_{1}, l_{2}}(d) \leqslant P_{3}(d)(1.209 \ldots)^{d}$, т.е. работа [92] снова дает лучшую оценку, чем работы [28] и [31].

4) Пусть, наконец, $d=24 d^{\prime}$, а $k=l_{1}=l_{2}=d / 24$. Тогда применение результатов работы [92] дает оценку $f_{k, l_{1}, l_{2}}(d) \leqslant P_{4}(d)(1.171 \ldots)^{d}$, которая опять-таки улучшает оценки из [28] и [31].

5) Однако, положив $d=4 d^{\prime}, k=d / 2, l=d / 2$, мы уже получим из [92] лишш оценку $f_{k, l}(d) \leqslant P_{5}(d)(1.240 \ldots)^{d}$, и эта оценка заведомо хуже, чем оценка из [28] или [31]. Аналогичньй пример можно привести и для кросс-политопов.

Заметим в заключение, что результаты работы [92] доказываются с использованием верхних оценок на мошность минимальной системы общих представителей, возникающей в задаче о покрытии (см. [93]-[101]), и что, кроме того, эти результаты непосредственно связаны с проблемой Турана в задаче о покрытии (см. [93], [95], [96], [102] и [103]). Отметим также, что некоторые вопросы, сходные с теми, что были изучены в [92], в частном случае рассматривались в [104]. И, наконец, сформулируем задачу.

ЗАДАЧА. Доказать гипотезу Борсука в каких-нибудь мальх размерностях для кросс-политопов.

Автор благодарит Г. М. Циглера за неоднократную помощь и поддержку, а также за любезно предоставленный им оттиск работы [91]. Наконец, автор особенно благодарен Н.Г. Мощевитину и Н.П. Долбилину за постоянный интерес и внимание к его работе.

\section{СПИСОК ЛИТЕРАТУРЫ}

[1] K. Borsuk. Drei Sätze über die $n$-dimensionale Euklidische Sphäre // Fund. Math. 1933. V. 20. P. $177-190$

[2] H. Hadwiger. Ein Überdeckungssatz für den Euklidischen Raum // Portugal. Math. 1944. V. 4. P. 140-144.

[3] K. Borsuk. Über die Zerlegung einer Euklidischen $n$-dimensionalen Vollkugel in $n$ Mengen // Verh. Internat. Math. Kongr., Zürich. 1932. V. 2. P. 192.

[4] В. Г. Болтянский, И. Ц. Гохберг. Теоремы и задачи комбинаторной геометрии. М.: Наука, 1965. 
[5] Л. А. Л юстерник, Л. Г. Шнирельман. Топологические методы в вариационных задачах. М.: МГУ, Иссл. ин-т матем. мех., 1930.

[6] H. Lenz. Zur Zerlegung von Punktmengen in solche kleineren Durchmessers // Arch. Math. 1955. V. 6. № 5. P. 413-416.

[7] И. М. Яглом, В. Г. Болтянский. Выпуклые фигуры. М.: Гостехиздат, 1951.

[8] J. Pál. Über ein elementares Variationsproblem. Copenhagen: Roy. Danish Acad. Sci. Letters, 1920. (Mat.-Fys. Medd. Danske Vid. Selsk. V. 3. № 2.)

[9] H. G. Eggleston. Covering a three-dimensional set with sets of smaller diameter // J. London Math. Soc. 1955. V. 30. P. 11-24.

[10] J. Perkal. Sur la subdivision des ensembles en parties de diamètre inférieur // Colloq. Math. 1947. V. 1. P. 45.

[11] B. Grünbaum. A simple proof of Borsuk's conjecture in three dimensions // Proc. Cambridge Philos. Soc. 1957. V. 53. P. 776-778.

[12] D. Gale. On inscribing $n$-dimensional sets in a regular $n$-simplex // Proc. Amer. Math. Soc. 1953. V. 4. P. 222-225.

[13] A. Heppes. Térbeli ponthalmazok felosztása kisebb átméröjü részhalmazok összegére // Magyar Tudományos Akadémia Mat. Fiz. Tud. Oszt. Közl. 1957. V. 7. P. 413-416.

[14] В. В. Макеев. Об афффинных образах ромбододекаэдра, описанных вокруг трехмерного выпуклого тела в $\mathbb{R}^{3}$ // Зап. науч. семин. ПОМИ. 1997. Т. 246. С. 191-195.

[15] В. В. Макеев. Аффинно-вписанные и аффинно-описанные многоугольники и многогранники // Зап. науч. семин. ПОМИ. 1996. Т. 231. С. 286-298.

[16] A. Heppes, P. Révész. Zum Borsukschen Zerteilungsproblem // Acta Math. Acad. Sci. Hungar. 1956. V. 7. P. 159-162.

[17] P. Erdös. On sets of distances of $n$ points // Amer. Math. Monthly. 1946. V. 53. P. 248-250.

[18] Г. Хадвигер, Г. Дебруннер. Комбинаторная геометрия плоскости. М.: Наука, 1965.

[19] P. Katzarowa-Karanowa. Über ein euklidisch-geometrisches Problem von B. Grünbaum // Arch. Math. 1967. V. 18. P. 663-672.

[20] Л. Данцер, Б. Грюнбаум, В. Кли. Теорема Хелли. М.: Мир, 1968.

[21] H. Hadwiger. Überdeckung einer Menge durch Mengen kleineren Durchmessers // Comment. Math. Helv. 1945/46. V. 18. P. 73-75; Mitteilung betreffend meine Note: Überdeckung einer Menge durch Mengen kleineren Durchmessers // Comment. Math. Helv. 1946/47. V. 19. P. 72-73.

[22] C. A. Rogers. Symmetrical sets of constant width and their partitions // Mathematika. 1971. V. 18. P. $105-111$.

[23] V. G. Boltyanski, H. Martini, P.S. Soltan. Excursions into combinatorial geometry. Berlin: Springer-Verlag, 1997.

[24] K. Borsuk. Some remarks on covering of bounded subsets of the Euclidean $n$-space with sets of smaller diameter // Demonstratio Math. 1978. V. 11. P. 247-251.

[25] M. Lassak. An estimate concerning Borsuk's partition problem // Bull. Polish Acad. Sci. Math. 1982. V. 30. P. 449-451.

[26] H.W. E. Jung. Über die kleinste Kugel, die eine räumliche Figur einschliesst // J. Reine Angew. Math. 1901. V. 123. P. 241-257.

[27] R. Knast. An approximative theorem for Borsuk's conjecture // Proc. Cambridge Philos. Soc. 1974. № 1. P. 75-76.

[28] O. Schramm. Illuminating sets of constant width // Mathematika. 1988. V. 35. P. $180-189$.

[29] В. Г. Болтянский. Задача об освещении границы выпуклого тела // Изв. Молдавского филиала АН СССР. 1960. № 10 (76). С. 77-84.

[30] H. G. Eggleston. Convexity. Cambridge: Cambridge Univ. Press, 1958.

[31] J. Bourgain, J. Lindenstrauss. On covering a set in $\mathbb{R}^{d}$ by balls of the same diameter // Geometric Aspects of Functional Analysis / ed. J. Lindenstrauss, V. Milman. Berlin: Springer-Verlag, 1991. P. 138-144. (Lecture Notes in Math. V. 1469.) 
[32] L. Danzer. On the $k$-th diameter in $E^{d}$ and a problem of Grünbaum // Proc. Colloquium on Convexity (Copenhagen, 1965), 1967. P. 41.

[33] C. A. Rogers. Covering a sphere with spheres // Mathematika. 1963. V. 10. P. 157-164.

[34] O. Schramm // M. Sci. thesis. Jerusalem: Hebrew University, 1987.

[35] P. Erdös. My Scottish book "problems" // The Scottish Book. Mathematics from the Scottish Café / ed. R. D. Mauldin. Basel: Birkhäuser, 1981. P. 35-43.

[36] C.A. Rogers. Some problems in the geometry of convex bodies // The Geometric Vein - The Coxeter Festschrift / ed. C. Davis, B. Grünbaum, F. A. Sherk. New York: Springer-Verlag, 1982. P. 279-284.

[37] D. Larman. Open problem 6 // Ann. Discrete Math. 1984. V. 20. P. 336.

[38] P. Frankl, R. Wilson. Intersection theorems with geometric consequences // Combinatorica. 1981. V. 1. P. 357-368.

[39] J. Kahn, G. Kalai. A counterexample to Borsuk's conjecture // Bull. Amer. Math. Soc. (N.S.). 1993. V. 29. № 1. P. 60-62.

[40] A. Nilli. On Borsuk's problem // Contemp. Math. 1994. V. 178. P. 209-210.

[41] J. Grey, B. Weissbach. Ein weiteres Gegenbeispiel zur Borsukschen Vermutung // Preprint № 25. Magdeburg: Univ. Magdeburg, Fakultät für Mathematik, 1997.

[42] А. М. Райгородский. О размерности в проблеме Борсука // УМН. 1997. Т. 52. № 6. C. $181-182$.

[43] B. Weissbach. Sets with large Borsuk number // Beiträge Algebra Geom. 2000. V. 41.

[44] А. М. Райгородский. Об одной оценке в проблеме Борсука // УМН. 1999. Т. 54. № 2. C. $185-186$.

[45] М. Л. Гервер. О разбиении множеств на части меньшего диаметра: теоремы и контрпримеры // Матем. просвещение (М.: МЦНМО, “ЧеРо"). Сер. 3. 1999. № 3. С. 168-183.

[46] M. Aigner, G. M. Ziegler. Proofs from THE BOOK. Berlin: Springer-Verlag, 1998.

[47] В. Г. Болтянский. О разбиении плоских фигур на части меньшего диаметра // Colloq. Math. 1970. T. 21. № 2. C. 253-263.

[48] В.Г. Болтянский, В.П. Солтан. Задача Борсука // Матем. заметки. 1977. Т. 22. № 5. C. 621-631.

[49] B. Grünbaum. Borsuk's problem and related questions // Proc. Sympos. Pure Math. 1963. V. 7. P. 271-284.

[50] H. Croft, K. Falconer, R. Guy. Unsolved Problems in Geometry. New York: Springer-Verlag, 1991.

[51] M. Benda, M. Perles. Colorings of metric spaces // не опубликовано.

[52] P.D. Johnson, Jr.. Introduction to "Colorings of metric spaces", by Benda and Perles // Geombinatorics. 2000. V. 9. P. 110-126.

[53] Ф. Харари. Теория графов. М.: Мир, 1973.

[54] N.G. de Bruijn, P. Erdös. A colour problem for infinite graphs and a problem in the theory of relations // Proc. Konink. Nederl. Akad. Wetensch. Ser. A. 1951. V. 54. № 5 . P. 371-373.

[55] R. Rado. Axiomatic treatment of rank in infinite sets // Canad. J. Math. 1949. V. 1. P. 337-343.

[56] L. A. Székely. Measurable chromatic number of geometric graphs and sets without some distances in Euclidean space // Combinatorica. 1984. V. 4. № 2-3. P. 213-218.

[57] Дж. Шенфилд. Математическая логика. М.: Наука, 1975.

[58] D. R. Woodall. Distances realized by sets covering the plane // J. Combin. Theory Ser. A. 1973. V. 14. P. 187-200.

[59] L. Moser, W. Moser. Solution to problem 10 // Canad. Math. Bull. 1961. V. 4. P. $187-189$.

[60] H. Hadwiger. Ungelöste Probleme № 40 // Elem. Math. 1961. V. 16. P. 103-104.

[61] Д. Е. Райский. Реализация всех расстояний при разбиении пространства $\mathbb{R}^{n}$ на $n+1$ часть // Матем. заметки. 1970. Т. 7. С. 319-323.

[62] D. Coulson. On 18-colouring of 3-space omitting distance one // Discrete Math. 1997. V. 170. P. 241-247. 
[63] K. B. Chilakamarri. On the chromatic number of rational five-space // Aequationes Math. 1990. V. 39. P. 146-148.

[64] K.B. Chilakamarri. The unit-distance graph problem: a brief survey and some new results // Bull. Inst. Combin. Appl. 1993. V. 8. P. 39-60.

[65] D. G. Larman, C. A. Rogers. The realization of distances within sets in Euclidean space // Mathematika. 1972. V. 19. P. 1-24.

[66] J. Zaks. On the four-colourings of rational four-space // Aequationes Math. 1989. V. 37. №2 2/3. P. 259-266.

[67] J. Zaks. On the chromatic numbers of some rational spaces // Ars Combin. 1992. V. 33. P. 253-256.

[68] L. A. Székely. Erdös on unit distances and the Szemerédi-Trotter theorems // Paul Erdös and his Mathematics. Bolyai Soc. Math. Stud., 2000.

[69] L. A. Székely. Erdös on unit distances and the Szemerédi-Trotter theorems // Paul Erdös and his Mathematics. Abstracts of invited talks held in the memory of Paul Erdös, Budapest, Hungary, July 4-11, 1999.

[70] D. G. Larman. A note on the realization of distances within sets in Euclidean space // Comment. Math. Helv. 1978. V. 53. P. 529-535.

[71] P. Frankl. Extremal problems and coverings of the space // European J. Combin. 1980. V. 1. P. 101-106.

[72] G. J. Butler. Simultaneous packing and covering in Euclidean space // Proc. London Math. Soc. (3), 1972. V. 25. № 4. P. 721-735.

[73] P. Erdös, C. A. Rogers. Covering space with convex bodies // Acta Arith. 1962. V. 7. P. 281-285.

[74] А. М. Райгородский. О хроматическом числе пространства // УМН. 2000. Т. 55. № 2. C. $147-148$.

[75] L. Babai, P. Frankl. Linear Algebra Methods in Combinatorics. Part 1. Preliminary version 2. Chicago: Department of Computer Science, The University of Chicago, 1992.

[76] V. Klee, S. Wagon. Old and New Unsolved Problems in Plane Geometry and Number Theory. Washington, DC: Math. Assoc. America, 1991.

[77] J. Pach, P. K. Agarwal. Combinatorial Geometry. New York: Wiley, 1995.

[78] A. Soifer. Chromatic number of the plane: A historical essay // Geombinatorics. 1991. V. 1. № 3. P. 13-15.

[79] A. Soifer. Mathematical Coloring Book. Colorado Springs: Center for Excellence in Mathematical Education, 1997.

[80] P. Erdös, Chao Ko, R. Rado. Intersection theorems for systems of finite sets // Quart. J. Math. Oxford Ser. (2). 1961. V. 12. P. 313-320.

[81] N. Alon, L. Babai, H. Suzuki. Multilinear polynomials and Frankl-Ray-ChaudhuriWilson type intersection theorems // J. Combin. Theory Ser. A. 1991. V. 58. P. 165-180.

[82] D. K. Ray-Chaudhuri, R. M. Wilson. On t-designs // Osaka J. Math. 1975. V. 12. P. $735-744$.

[83] M. Deza, P. Erdös, P. Frankl. Intersection properties of systems of finite sets // Proc. London Math. Soc. (3). 1978. V. 36. P. 369-384.

[84] M. Deza, P. Erdös, N. M. Singhi. Combinatorial problems on subsets and their intersections // Adv. Math., Suppl. Stud. 1978. V. 1. P. 259-265.

[85] К. Прахар. Распределение простых чисел. М.: Мир, 1967.

[86] A. M. Raigorodskii. On two combinatorial problems // Paul Erdös and his Mathematics. Abstracts of talks held in the memory of Paul Erdös, Budapest, Hungary, July 4-11, 1999.

[87] F. Schiller. Zur Berechnung und Abschätzung von Färbungszahlen und der $\vartheta$-Funktion von Graphen // Diplomarbeit. Berlin: Technische Universität, 1999.

[88] C. Payan. On the chromatic number of cube-like graphs // Discrete Math. 1992. V. 103. P. 271-277.

[89] J. Petersen. Färbung von Borsuk-Graphen in niedriger Dimension // Diplomarbeit. Berlin: Technische Universität, 1998.

[90] G. M. Ziegler. Lectures on 0/1-polytopes // DMV Sem. 2000. V. 29. P. 1-44. 
[91] G. M. Ziegler. Coloring Hamming graphs, optimal binary codes, and the 0/1-Borsuk problem in low dimensions // Preprint. Berlin: Technische Universität, 2000.

[92] А. М. Райгородский. Проблема Борсука для $(0,1)$-многогранников и кросс-политопов // Докл. РАН. 2000. Т. 371. С. 600-603.

[93] П. Эрдеш, Дж. Спенсер. Вероятностные методы в комбинаторике. М.: Мир, 1976.

[94] N. Alon, J. Spencer. The Probabilistic Method. New York: Wiley, 1992.

[95] В. Е. Тараканов. Комбинаторные задачи и $(0,1)$-матрицы. М.: Наука, 1985.

[96] Н.Н. Кузюрин. Асимптотическое исследование задачи о покрытии // Проблемы кибернетики. 1980. №37. С. 19-56.

[97] В.К. Леонтьев. Верхние оценки $\alpha$-глубины $(0,1)$-матриц // Матем. заметки. 1974. T. 15. №3. C. 421-429.

[98] Э.И. Нечипорук. О топологических принципах самокорректирования // Проблемш кибернетики. 1969. № 21. С. 5-103.

[99] А. А. Сапоженко. О сложности дизъюнктных нормальных форм, получаемшіх с помощью градиентного алгоритма // Дискретн. анализ (Новосибирск). 1972. № 21. С. 62-71.

[100] Е. Д. Глускин. Экстремальные свойства ортогональных параллелепипедов и их приложение к геометрии банаховых пространств // Матем. сб. 1988. Т. 136. № 1. С. 85-96.

[101] А. М. Райгородский. Системы общих представителей // Фунд. прикл. матем. 1999. T. 5. № 3. C. $851-860$.

[102] P. Turan. Egy grafelmeletiszelsoertek feladatrol // Mat. Fiz. Lapok. 1941. V. 48. P. $436-452$.

[103] G. Katona, T. Nemetz, M. Simonovitz. On a graph problem of Turan // Mat. Lapok. 1964. V. 15. P. 228-237. (Hungarian.)

[104] N. Linial, R. Meshulam, M. Tarsi. Matroidal bijections between graphs // J. Combin. Theory Ser. B. 1988. V. 45. № 1. P. 31-44.

Московский государственньй

Поступила в редакцию

университет им. М. В. Ломоносова

07.12 .2000 